\title{
Die Einbindung zivilgesellschaftlicher Akteure in die Härtefallkommissionen der Bundesländer: Bandbreite, Ursachen, Folgen*
}

\section{Kurzfassung}

Mit dem Zuwanderungsgesetz der rot-grünen Bundesregierung von 2004 bekamen die Bundesländer die Möglichkeit, Härtefallkommissionen einzurichten, welche aus humanitären Gründen die Innenministerien um die Erteilung einer Aufenthaltserlaubnis an ausreisepflichtige Ausländer ersuchen können. Alle Bundesländer haben solche Härtefallkommissionen (HFKs) in der Zwischenzeit eingerichtet, und in 15 Bundesländern sind auch zivilgesellschaftliche Akteure darin vertreten.

Der Artikel untersucht die zwischen den Bundesländern feststellbare Bandbreite dieser Beteiligung in den Härtefallkommissionen auf ihre Ursachen und Folgen. $\mathrm{Zu}$ den wichtigsten Ursachen zählen parteipolitische Unterschiede in der Regierungszusammensetzung. Bei den Folgen sind die stark unterschiedlichen Ersuchens-, Stattgabe- und Erfolgsquoten, die auch auf weitere Determinanten hin untersucht werden, aber stärker idiosynkratisch bleiben, sowie der potenzielle Identitäts- und Legitimationskonflikt auf der Seite der zivilgesellschaftlichen Akteure zuvorderst zu nennen. Im Fazit wird neben einigen Lehren zur zukünftigen Praxis der staatlichen wie der nichtstaatlichen Seite die Frage beantwortet, ob die HFKs in ihrer gegenwärtigen Form eher als Feigenblatt oder als i-Tüpfelchen der Politik des humanitären Aufenthaltsrechts angesehen werden können.

* Der Autor dankt den beiden anonymen Gutachtern und der Redaktion der ZPol sowie den Teilnehmern des Workshops „Legitimationspraktiken als Integrationspolitik“ im Rahmen der Dreiländertagung von SVPW, ÖGPW und DVPW am 13./14. Januar 2011 in Basel für zahlreiche hilfreiche Kommentare und Verbesserungsvorschläge. 


\section{Inhalt}

1. Einleitung 260

2. Einrichtung, Besetzung und rechtliche Vorgaben der Härtefallkommissionen

3. Folgen der Einbindung zivilgesellschaftlicher Akteure in die Härtefallkommissionen: Entscheidungspraxis und Legitimationsfragen $\quad 270$

a) Entscheidungsstatistik und Fallauswahl 270

b) Qualitative Analyse der Entscheidungspraxis 273

c) Quantifizierende Analyse der Entscheidungspraxis 279

d) Identitäts- und Legitimationskonflikte auf Seiten der Nichtregierungsorganisationen 283

4. Fazit: Feigenblatt oder i-Tüpfelchen? 286

\section{Einleitung}

Während die „Ausdifferenzierung der Migrationspolitik [in] Anwerbung der , besten Köpfe“ und Flüchtlingsabwehr" (Butterwegge 2009: 74) in Deutschland im Hinblick auf Erstere noch nicht recht in Fahrt kommt, ist Letztere längst etabliert. Der sogenannte Asylkompromiss sorgte bereits 1994 - unter anderem mit der Drittstaatenregelung, der Positivliste angeblich sicherer Herkunftsländer und dem verkürzten Flughafenverfahren - für nur noch sehr geringe Chancen auf politisches Asyl in Deutschland und kann als Teil einer ,restriktive[n] Wende in der Asylpolitik der entwickelten Industriestaaten“ (Holzer/Schneider 2002: 17) interpretiert werden. ${ }^{1}$ In der Folge wuchs die Zahl der Härtefälle unter den abgelehnten Bewerbern an, und nach dem Ende der Kriege im ehemaligen Jugoslawien kamen Zehntausende ausreisepflichtige Flüchtlinge hinzu, die eine Rückkehr als unzumutbar empfanden. Verschiedene zivilgesellschaftliche Akteure wie Kirchen, Wohlfahrtsverbände und insbesondere einige einschlägige Nichtregierungsorganisationen (NROs) kümmerten sich von Beginn an um diese Menschen und formulierten politische Initiativen zu ihren Gunsten.

1 Angesichts der im Industrieländervergleich extrem hohen Asylbewerberzahlen von insgesamt über einer Million zwischen 1991 und 1993 war eine Anpassungsreaktion Deutschlands nicht überraschend. Ob dafür das Kind mit dem Bade ausgeschüttet werden musste, sei dahingestellt. Der Druck auf das Asylsystem indes ist nicht zu verstehen, ohne die Reglementierung der Arbeitsmigration in den Blick zu nehmen (zu den arbeitsmarkt- und identitätspolitischen Determinanten Letzterer vgl. Cable 1995: 39). 
Nach anfänglich überwiegender Abwehrhaltung von staatlicher Seite kam es mit dem rot-grünen Zuwanderungsgesetz zu einer institutionellen Innovation, die zumindest einer Teilgruppe der ausreisepflichtigen Ausländer eine Bleibeperspektive eröffnet und die Einbindung zivilgesellschaftlicher Akteure in die Asyl- und Flüchtlingspolitik der Bundesländer ermöglicht: Paragraph 23 a des als Teil des Zuwanderungsgesetzes 2004 verabschiedeten Aufenthaltsgesetzes (AufenthG) erlaubt den Bundesländern die Einrichtung von Härtefallkommissionen (HFKs) ${ }^{2}$ und den obersten Landesbehörden die Erteilung einer Aufenthaltserlaubnis, wenn die Kommission sie darum ersucht. ${ }^{3}$

Alle Bundesländer haben solche Härtefallkommissionen in der Zwischenzeit eingerichtet, und in allen 15 Bundesländern außer Hamburg sind derzeit auch zivilgesellschaftliche Akteure darin vertreten. Nordrhein-Westfalen ist das einzige Land, das die von $\S 23$ a (2) AufenthG eröffnete Möglichkeit nutzt, die Kompetenz zur Anordnung der Aufenthaltserlaubnis - vulgo das Veto gegen die Empfehlungen der HFK - einer anderen Stelle als der obersten Landesbehörde zu übertragen, in diesem Fall den kommunalen Ausländerbehörden, die in aller Regel auch für die Erteilung von Aufenthaltstiteln auf Basis anderer gesetzlicher Grundlagen zuständig sind.

Die Härtefallkommissionen stellen nicht nur eine „Melange aus säkularisiertem Kirchenasyl und Gnadenrecht ${ }^{\star 4}$ dar, sie bilden auch ein Prisma von vier politischen Ebenen: Auf Landesebene verankert ergeben sich ihre Agenden zum Großteil aus bundes- und europarechtlichen Rahmenbedingungen, wohingegen sich eine Politisierung der von ihr behandelten Fälle nahezu ausnahmslos auf der lokalpolitischen Ebene abspielt. Ihre Praxis, so wird im Weiteren detaillierter zu zeigen sein, variiert im Hinblick auf die zur Beratung zugelassenen Anträge, die Häufigkeit der beschlossenen Ersuchen um Erteilung einer Aufenthaltserlaubnis und die Verbreitung

2 Die Härtefallkommissionen verfügen in der Regel zwar über Entscheidungsgrundsätze, nicht aber über eine Arbeitsdefinition des Begriffs Härtefall. Das Aufenthaltsgesetz spricht allgemein von „dringende[n] humanitäre[n] oder persönliche[n] Gründen“ (§ 23 a (2) AufenthG).

3 Wohlgemerkt: Ohne ein Ersuchen der Härtefallkommissionen (und wie wir noch sehen werden, kann in 14 Bundesländern die staatliche Seite in der HFK allein kein Ersuchen herbeiführen) kann das Land die Ermächtigung des $\S 23$ a AufenthG zur Erteilung einer Aufenthaltserlaubnis aus humanitären Gründen nicht nutzen. Das Aufenthaltsgesetz kennt auch andere, über verschiedene Paragraphen verstreute Ausnahmeregelungen, die die Länder zur Erteilung von Aufenthaltstiteln ermächtigen. Diese sind indes an einzeln aufgeführte, konkrete Tatbestände gebunden und ermöglichen gerade nicht eine gnadenrechtliche, dem Rechtsweg enthobene Gesamtabwägung wie $\S 23$ a (vgl. hierzu auch Wais 2010: 254).

4 Zitat von Dr. Ralph Göbel-Zimmermann, Vorsitzender Richter am Verwaltungsgericht Wiesbaden, aus dem Stenographischen Bericht zur Anhörung des Innenausschusses des hessischen Landtages am 24.9.2009. 
einstimmiger Beschlüsse ebenso stark wie die Neigung der obersten Landesbehörden, den HFK-Ersuchen stattzugeben.

Der vorliegende Artikel beschreibt vor diesem Hintergrund zunächst die Einrichtung und Besetzung der Härtefallkommissionen sowie die rechtlichen Vorgaben für ihre Arbeit im Detail (Kap. 2). Sodann untersucht er die Bandbreite der Beteiligung nichtstaatlicher Akteure in HFKs in den Ländern auf ihre Folgen (Kap. 3). Hier geht es neben der Analyse der Entscheidungsstatistik sowohl um eine kritische Bilanz der ersten fünf Jahre HFK-Aktivität als auch um eine Durchleuchtung der Motive, aus denen die Landesregierungen und -parlamente die zivilgesellschaftlichen Vertreter eigentlich kooptieren - und aus denen diese sich zur Mitwirkung entscheiden..$^{5}$ Im Fazit (Kap. 4) folgen auf eine Einschätzung, ob die Härtefallkommissionen eher Feigenblatt eines restriktiven humanitären Aufenthaltsrechts oder das i-Tüpfelchen auf den rot-grünen Erleichterungen desselben sind, auch einige Bemerkungen zu Lehren für die künftige Praxis von Politik und zivilgesellschaftlichen Akteuren sowie die politikwissenschaftliche Erforschung derselben.

Zugrunde liegt dem Artikel ein methodenverbindendes Forschungsdesign. Für die quantifizierenden Analysen wurde die Bundesländer-Vergleichsdatenbank des Verfassers mit einer von Andreas Schwantner von der deutschen Sektion von Amnesty International erstellten Synopse der HFK-Strukturen und -Fallstatistiken kombiniert. Auf der qualitativen Seite stehen neben der Auswertung von umfangreichen Primärtextquellen ${ }^{6}$ vierzehn teilstandardisierte Experteninterviews mit Mitgliedern der HFKs und weiteren Akteuren in deren administrativem wie zivilgesellschaftlichem Umfeld ${ }^{7}$ in fünf Bundesländern sowie zwei teilnehmende Beobachtungen an

5 Das Selbstbild der NROs kann mit dieser Mitwirkung konfligieren, aber im Laufe der Beteiligung auch symbiotisch adaptiert werden.

6 Hierzu zählen insbesondere die HFK-Gesetze und -Verordnungen der Bundesländer, parlamentarische Protokolle aus der Zeit der Vorbereitung und Verabschiedung derselben, die von einer Mehrzahl der Kommissionen vorgelegten und auf der jeweiligen Internetpräsenz veröffentlichten Tätigkeitsberichte sowie graue Literatur aus dem nichtstaatlichen Umfeld der HFKs. Diese Quellen werden aus Platzgründen nicht in einem eigenen Verzeichnis aufgeführt, sondern ins allgemeine Literaturverzeichnis aufgenommen, sofern wörtlich daraus zitiert wird.

7 Unter den Gesprächspartnern waren Vertreter der staatlichen (Mitarbeiter der Innenministerien, Ausländerbehörden und von kommunalen Verbänden, Parlamentsabgeordnete sowie staatlicherseits nominierte Pensionäre) wie der nichtstaatlichen Seite (hier von Kirchen, Wohlfahrtsverbänden, Flüchtlingsräten, Pro Asyl, Amnesty International und der Ärzteschaft) in HFKs, außerdem neben ordentlichen Mitgliedern auch zwei HFK-Vorsitzende. Die Zitation der Aussagen aus den 30-minütigen bis dreistündigen Gesprächen erfolgt aus Rücksicht auf die Gesprächspartner anonym, im generischen Maskulinum und in der Regel indirekt. Eine breite Streuung der Gesprächspartner 
HFK-Sitzungen. Letztere dienten insbesondere der Validierung von aus anderen Quellen gewonnenen Vorverständnissen. ${ }^{8}$

Den theoretischen Hintergrund des Artikels bilden vornehmlich die kanonisierten Ansätze der vergleichenden Staatstätigkeitsforschung (vgl. Schmidt u. a. 2007; zu ihrer Anwendung auf privates Handeln Wolf/Zohlnhöfer 2009: 231 ff.), wobei parteipolitische, interessengruppenbezogene, institutionelle und sozioökonomische Faktoren besondere Aufmerksamkeit genossen. Auf einen ausformulierten Hypothesenkatalog wurde für die Zwecke dieses eher induktiv-explorativ Neuland erkundenden Vorhabens indes bewusst verzichtet.

Überdies steht die Befassung mit der Rolle zivilgesellschaftlicher Akteure im Lichte der Governance-Literatur. Allerdings geht es hier nicht um die Kernelemente des Governance-Begriffs wie des „Managements von Interdependenzen“ und der „Kombination aus unterschiedlichen Regelsystemen“ (Benz 2004: 25), sondern um die Indienstnahme zivilgesellschaftlicher Kompetenz und Legitimationsbeiträge für staatliches Problemlösungshandeln.

\section{Einrichtung, Besetzung und rechtliche Vorgaben der Härtefallkommissio- nen}

Aus Tabelle 1 gehen die Gründungsdaten der Härtefallkommissionen, die parteipolitische Färbung der jeweiligen Landesregierung zum entsprechenden Zeitpunkt und die Besetzung der Kommissionen hervor. Bremen und Hessen haben die Besetzung bereits ein- bzw. zweimal revidiert, weshalb sie mehrere Zeilen erhalten. In manchen Ländern (u. a. Nordrhein-Westfalen ${ }^{9}$ und Berlin) gab es bereits vor der Verabschiedung des Aufenthaltsgesetzes gleichnamige Kommissionen mit anderen Kompetenzen. Diese sind nicht berücksichtigt; zuweilen wurden indes durch die HFK-Verordnungen oder -Gesetze die existierenden Kommissionen in identischer Besetzung neu konstituiert, was sich auf Arbeitsweise und -klima auswirken konnte.

konnte durchaus erreicht werden, perfekte Repräsentativität über die verschiedenen Variationsdimensionen hingegen nicht. Dies liegt unter anderen daran, dass die Bereitschaft zu einem Gespräch auf der staatlichen Seite geringer ausgeprägt war. Bei der Interpretation und Gewichtung der Interviewaussagen wurde selbstverständlich alles daran gesetzt, aus dieser Asymmetrie womöglich folgende Einseitigkeiten zu vermeiden.

8 Ausführlicheres zur Fallauswahl und den zur Anwendung gebrachten Methoden folgt in Kap. 3. Zum besonderen Reiz wie zu Schwierigkeiten bundesländervergleichender Forschungsdesigns vgl. im Übrigen Hildebrandt/Wolf 2008.

9 Zur Arbeit dieser Vorläuferkommission, die in einem Interview auch als entscheidendes Vorbild für die § 23a-Regelung bezeichnet wurde, vgl. Weber 2005. 
Frieder Wolf

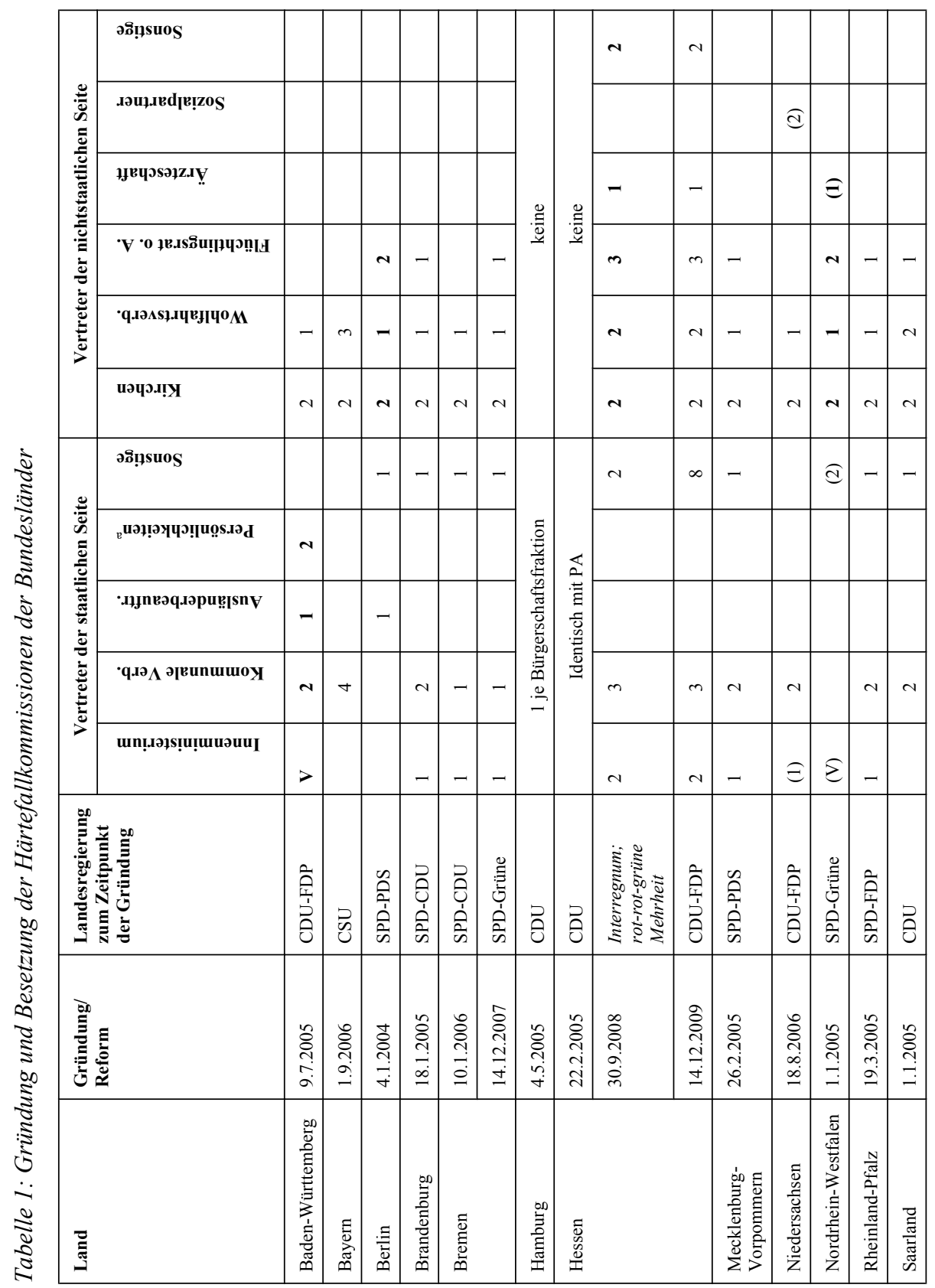




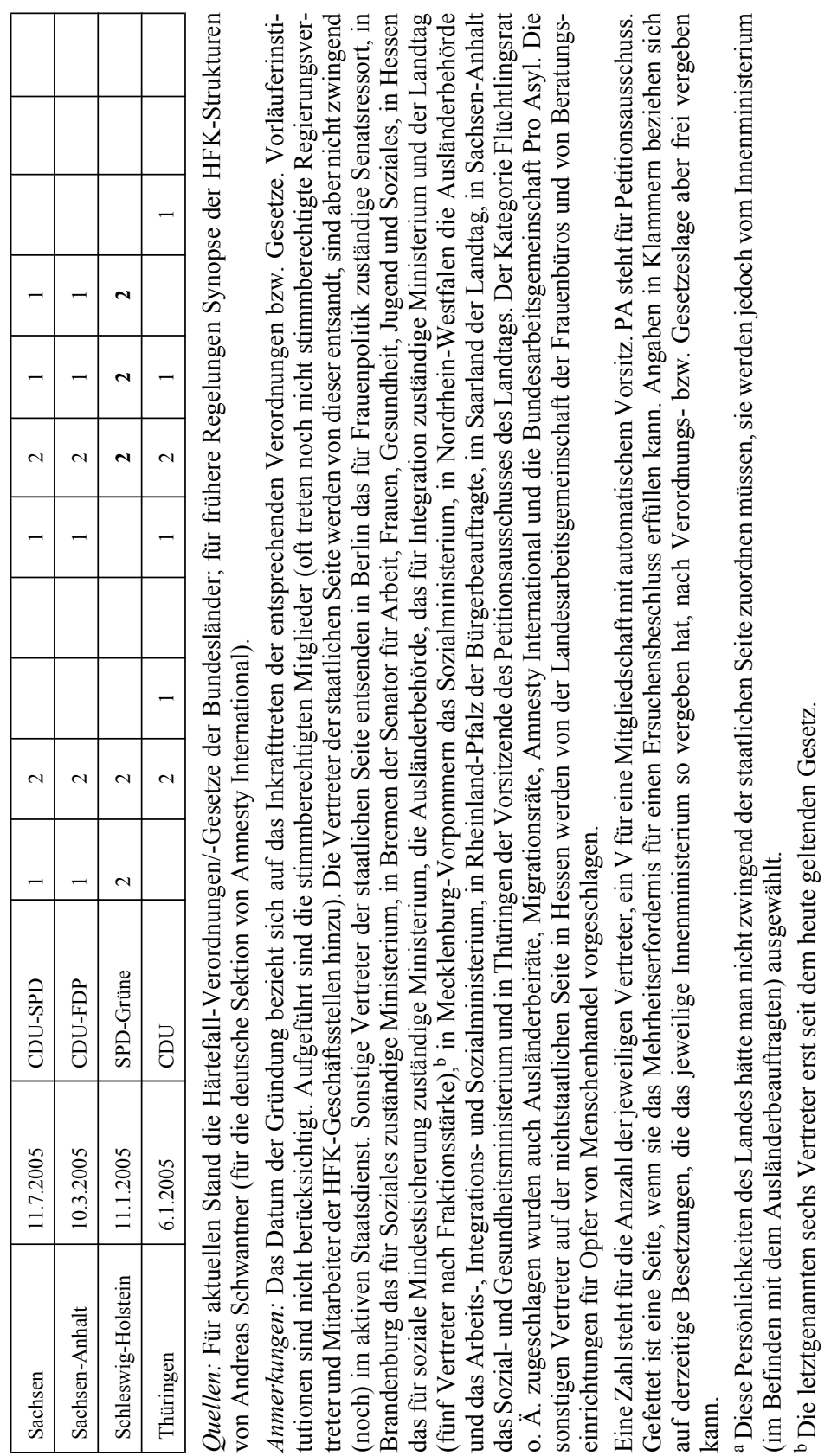


Auffallend ist, dass fast alle linken Landesregierungen (und nur solche) den Vertretern der Zivilgesellschaft eine einfache Mehrheit zugestehen, und dass immerhin vier von sechs Linksregierungen inklusive der linken Mehrheit in Hessen, die zwar das entsprechende Gesetz änderte, aber keine Regierung zustande brachte, es im Zusammenspiel von HFK-Besetzung und Mehrheitserfordernis den nichtstaatlichen Akteuren ermöglichen, ein Ersuchen gegen die Stimmen der staatlichen Seite zu beschließen. Zweidrittelmehrheiten sind in einer Minderheit der von linken Regierungen eingerichteten Härtefallkommissionen erforderlich, aber in einer Mehrheit aller HFKs.

Die bürgerlichen Regierungen setzen tendenziell höhere Mehrheitsschwellen voraus und statten die nichtstaatliche Seite seltener mit einer einfachen Mehrheit aus, aber nur in einem Fall ermöglichen sie es den staatlichen Vertretern, die zivilgesellschaftlichen Akteure zu überstimmen. ${ }^{10}$ Große Koalitionen schließlich setzen durchweg auf paritätische Besetzungen der HFKs. Die Bandbreite der vertretenen NROs ist bei von Linksregierungen eingesetzten HFKs im Durchschnitt größer; an allen Landesregierungen, die keine Flüchtlingsorganisationen berufen haben, ist die Union beteiligt. ${ }^{11}$ Schon bei der Bestallung der Kommissionen können wir also klare Parteiendifferenzen (bzw. genauer: Koalitionsdifferenzen) konstatieren. Wie sich diese Unterschiede auf die Ersuchenspraxis der HFKs und die bei den obersten Landesbehörden erreichten Stattgabequoten auswirken, bleiben zwei der wichtigsten Fragen für die nachfolgenden Analysen.

Zuvor aber sind noch einige Regelungen aus den HFK-Gesetzen bzw. -Verordnungen der Bundesländer zu erläutern, die für die Entscheidungspraxis hochrelevant sind. Das Aufenthaltsgesetz überlässt die Verfahrensregelungen ${ }^{12}$ sowie die Formulierung von Ausschlussgründen den Landesgesetzgebern. Es benennt jedoch explizit die Möglichkeit, dass die Sicherung des Lebensunterhalts durch die Antragsteller selbst oder Verpflichtungserklärungen Dritter berücksichtigt werden, und es erklärt die Annahme eines Härtefalls für in der Regel ausgeschlossen, „wenn der

10 In der Praxis, so wird bei der näheren Analyse dieses Falls zu sehen sein, spielt dies in BadenWürttemberg aber kaum eine Rolle.

11 Die einzigen beiden Fälle von Kommissionen ohne Beteiligung zivilgesellschaftlicher Akteure gehen auf CDU-Alleinregierungen zurück. In Hessen ist dies mittlerweile Geschichte, in Hamburg konnten die Grünen in der zwischenzeitlichen schwarz-grünen Koalition ihren Wunsch nach Umbesetzung nicht durchsetzen. (Darüber, ob der Verzicht der beiden CDU-Alleinregierungen auf kirchliche Vertreter etwas über die relative Kirchenferne der entsprechenden Landesverbände aussagt, kann hier nur spekuliert werden.)

12 Eine Übersicht bietet eine unter www.fluechtlingsinfo-berlin.de/fr/pdf/HFK_Laenderuebersicht.pdf (letzter Zugriff: 29.8.2011) verfügbare Synopse, die von Andreas Schwantner (Deutsche Sektion von Amnesty International) erstellt wurde. 
Ausländer Straftaten von erheblichem Gewicht begangen hat" (§23a (2) AufenthG). In den landesrechtlichen Regelungen sind Nichtbefassungs- und Regelausschlussgründe zu unterscheiden: Erstere führen dazu, dass ein Antrag überhaupt nicht behandelt wird, letztere in der Regel - aber, wie in Kapitel 3 zu sehen sein wird, eben nicht immer - zu einer negativen Entscheidung der HFK. Aus der Bandbreite der einschlägigen landesrechtlichen Regelungen (für eine Übersicht vgl. Allenberg 2010) ${ }^{13}$ erwächst eine ganz erhebliche Variation der Aussichten auf Befassung mit einem konkreten Härtefallantrag und seine Annahme. So ist etwa der zeitweise Bezug auch nur ergänzender Sozialleistungen in Bayern bereits ein K.O.Kriterium, und mehrere Länder lassen keine Befassung mehr zu, wenn die Flüchtlinge (auch nur zeitweise) ihre Identität verschleiert bzw. bei der Passbeschaffung nicht kooperiert haben - und das in einem System, in dem man zumindest nach Ansicht vieler interviewter Zivilgesellschaftsvertreter ,mit der Wahrheit oft nicht weiterkommt" (Zitat aus einem Experteninterview mit einem HFK-Mitglied). ${ }^{14}$ Es kann zudem vorkommen, dass ganze Familien chancenlos sind, wenn bei einem einzelnen Mitglied die betreffenden Nichtbefassungs- oder Regelausschlussgründe vorliegen; insbesondere müssen sich minderjährige Kinder oft de facto das Verhalten ihrer Eltern anrechnen lassen.

Herkunftslandbezogene Sachverhalte, die im Asylverfahren und/oder bei der Entscheidung über eine Duldung ${ }^{15}$ bereits thematisiert wurden, sollen in fast allen Bundesländern nicht noch einmal von den HFKs berücksichtigt werden. Oft fließen sie mehreren Expertengesprächen zufolge indes durchaus in die Gesamtabwägung der absoluten und relativen Perspektiven von Antragstellern ein - nicht zuletzt auch deshalb, weil insbesondere, aber nicht ausschließlich nichtstaatliche Mitglieder die Inhalte der entsprechenden Lageberichte zu verschiedenen Ländern als geschönt ansehen. Ähnliches gilt dort, wo Ärztevertreter in den HFKs sitzen, auch für medizinische Abschiebehindernisse. Nicht ohne Grund: Eine Untersuchung der Psychologischen Forschungs- und Modellambulanz für Flüchtlinge der Universität Konstanz kam zu dem Ergebnis, dass 40\% der Asylbewerber unter posttraumatischen Belastungsstörungen leiden und diese im Verfahren meist nicht erkannt, geschweige denn gewürdigt werden (vgl. Gäbel u. a. 2006). Während von der staatlichen Seite

13 Eine etwas anders angeordnete, ergänzte und aktualisierte Fassung, die der quantifizierenden Analyse dieses Erklärungsfaktors in Kapitel $3 \mathrm{c}$ zugrunde liegt, ist beim Autoren erhältlich.

14 Flüchtlinge sehen sich oft gezwungen, ihre wahre Identität zu verschleiern. Dazu tragen fragwürdige Anreize hiesiger Gesetze und Praktiken ebenso bei wie Ängste vor bei der Rückkehr drohenden Szenarien. Ihr Überlebenskampf wird damit auch einer um ,the possibility of fixing a sovereign subjectivity" (Ruback 2010: 491).

15 Technisch gesprochen wird hier das Vorliegen von Abschiebungshindernissen geprüft, in der Regel durch die kommunalen Ausländerämter. 
in manchen Ländern eine Vertretung der Ärzteschaft in der HFK als unangemessen abgelehnt wird, wird sie von anderen als segensreich betrachtet, wenn auch die Berücksichtigung ihrer Expertise rechtlich betrachtet in eine „Grauzone zwischen Transportfähigkeit und zielstaatsbezogenen Argumenten" (ein staatliches HFKMitglied) fällt.

Auf der Arbeitsebene haben sich viele Härtefallkommissionen Geschäftsordnungen oder Verfahrensgrundsätze gegeben, die das Tagesgeschäft strukturieren. Das in $\S 23$ a AufenthG im Grundsatz festgeschriebene Selbstbefassungsprinzip erfährt seine Konkretisierung mancherorts erst auf dieser Ebene; zuweilen ist der konkrete Selbstbefassungsmechanismus zumindest in seinen Details aber auch gar nicht schriftlich fixiert. Diese Ausgestaltung ist nicht ohne Belang für die Chancen der Antragsteller. So kann es etwa sein, dass der Vorsitzende alle Anträge zumindest nachrichtlich in die Kommission einbringt (wie etwa in Baden-Württemberg, wo bei Widerspruch anderer Mitglieder dann auch die vom Vorsitzenden zur Nichtbehandlung vorgesehenen Fälle beraten werden), es kann aber auch vorkommen, dass ein Antrag bis zum Fristablauf in der Geschäftsstelle liegt, weil sich kein Mitglied findet, das ihn der Kommission vorstellt (so zeitweise in Hessen). Manche Länder kennen - gewissermaßen als Zwischenlösung zwischen Kontrolle des Vorsitzenden und gleichberechtigtem ,Sushi-Bar-Prinzip“ (Anträge gehen allen Mitgliedern zu bzw. liegen für sie in der HFK-Geschäftsstelle aus und können von diesen eingebracht werden oder eben auch nicht) - auch Vorprüfungsausschüsse. In Letzteren reicht meist ein von den Zivilgesellschaftsvertretern auslösbares Minderheitenvotum aus, damit ein Fall der gesamten HFK vorgelegt wird. In einigen Ländern, etwa Berlin, Niedersachsen, dem Saarland und Sachsen, laufen die Anträge nicht bei der Geschäftsstelle ein und stehen den Mitgliedern über diese zur Verfügung, sondern sie sind qua Gesetz bzw. Verordnung direkt an einzelne Mitglieder zu richten, welche diese ohne Verfahrens- oder Begründungspflichten in die HFKs einbringen oder ignorieren können. ${ }^{16}$ Nordrhein-Westfalen hebt sich in dieser Hinsicht von den übrigen genauer analysierten Fällen insofern ab, als die einzelnen Anträge in den Sitzungen weder vom Vorsitz noch von anderen Mitgliedern einführend zusammengefasst, sondern ohne Präludium sofort diskutiert werden.

In Hamburg fungiert in gewisser Weise der - dort als noch selbstbewusster als andernorts geltende - Eingabeausschuss (vulgo: Petitionsausschuss) als funktiona-

16 Aus einem dieser Bundesländer ist das Gerücht zu vernehmen, dass Anwaltskanzleien gegen höhere dreistellige Beträge anbieten, als aussichtsreich angepriesene Kontakte zu HFK-Mitgliedern herzustellen. Ganz unabhängig vom Wahrheitsgehalt dieser angeblichen Vorkommnisse ist eine gnadenrechtliche Institution mit Selbstbefassungsprinzip durch einzelne Mitglieder natürlich hochgradig von der Integrität der Mitglieder abhängig, wenn Willkür vermieden werden soll. 
les Äquivalent zu einem Vorprüfungsausschuss, da die HFK nicht direkt, sondern nur nach vorheriger Eingabe erreicht werden kann. Die HFK-Mitglieder sitzen alle auch im Eingabeausschuss und beeinflussen die Weitergabe maßgeblich, sodass de facto eine Vorsortierung nach Passung bzw. Erfolgsaussichten stattfindet. Aber auch wo das Petitionsverfahren nicht zwingend vorgeschaltet ist (wie neben Hamburg auch in Hessen), haben vom Petitionsausschuss angeregte Härtefallanträge besonderes Gewicht bzw. besonders gute Erfolgsaussichten. ${ }^{17}$ Zum nicht immer einfachen Verhältnis zwischen Härtefallkommission und Petitionsausschuss bemerkt ein HFK-Vorsitzender im Interview: „,Der Petitionsausschuss hat ganz andere Möglichkeiten und Erfolgsaussichten als die HFK, Vorschläge zur Änderung der generellen Politik- und Verwaltungspraxis zu machen. Von daher ist hier produktiver Austausch sinnvoller als eifersüchtiges Konkurrenzgebaren."

Erhält ein Antragsteller schließlich eine Aufenthaltserlaubnis, ist diese in der Regel zunächst auf drei Jahre befristet. Die Verfahren zur Verlängerung durch die Ausländerbehörden sind wiederum zwischen den Bundesländern unterschiedlich ausgestaltet. Erneute Befassungen der HFKs sind nirgends vorgesehen, teils aber ihre Information bei Nichtverlängerung (vgl. Allenberg 2010: $14 \mathrm{ff}$.). Die HFKs können jedoch in einigen Fällen selbst Bedingungen für eine spätere Verlängerung formulieren (ebd.: 15). Über das Entstehen neuer Härten in diesem Rahmen war den darauf angesprochenen Gesprächspartnern bislang nichts bekannt, auszuschließen sind sie indes nicht.

Bleibt die Frage, warum überhaupt alle Bundesländer die von $\S 23$ a AufenthG eröffnete Möglichkeit zur Einrichtung einer Härtefallkommission genutzt haben. Vieles spricht für eine Kombination aus politischen Diffusions- und Lernprozessen einerseits, über welche allerdings abgesehen von einigen Hinweisen auf informelle Kontakte in Experteninterviews an dieser Stelle nur spekuliert werden kann, ${ }^{18}$ mit der Anziehungskraft der Option zur ,blame-avoidance', die diese institutionelle Innovation bietet, andererseits. Letzten Endes dürfte überdies der politische Druck insbesondere von der kommunalen Ebene auf Nachzügler wie Bayern und Niedersachsen höher gewesen sein als die Kosten der HFK-Einrichtung.

17 Die Bayerische Verordnung spricht vom „Vorrang des Petitionsverfahrens“, wonach nach Durchlaufen eines Petitionsverfahrens ein Härtefallantrag nur noch möglich ist, wenn der Petitionsausschuss eine Weiterleitung beschließt. Eine Petition nach abgeschlossenem HFK-Verfahren ist dagegen möglich. Laut Tätigkeitsbericht für 2008 (Geschäftsstelle der Härtefallkommission in Bayern 2009: 6) soll damit der „Vorrang des Landtags“ sichergestellt werden.

18 War die grundsätzliche Entscheidung für die Einrichtung einmal getroffen, gab es bzgl. ihrer konkreten Gestalt indes in mehreren Ländern Anhörungen von beteiligten Akteuren aus anderen Bundesländern. 


\section{Folgen der Einbindung zivilgesellschaftlicher Akteure in die Härtefallkom- missionen: Entscheidungspraxis und Legitimationsfragen}

Um zu überprüfen, ob sich die bis hierher diskutierten Muster der Kooptation in die Härtefallkommissionen auf systematische oder zumindest charakteristische Weise in deren Entscheidungspraxis niederschlagen, ist diese zunächst näher zu beschreiben und dann sowohl qualitativ als auch quantifizierend zu analysieren. Gedanken zu Legitimations- und letzten Endes Identitätsfragen auf der nichtstaatlichen Seite und hier insbesondere bei den Nichtregierungsorganisationen folgen in einem eigenen Unterkapitel.

\section{a) Entscheidungsstatistik und Fallauswahl}

Wie aus Tabelle 2 hervorgeht, waren bis Ende 2009 etwas mehr als 2200 Fälle erfolgreich, sodass 5000 Menschen ein sonst nicht erreichbares Bleiberecht zugesprochen bekamen. ${ }^{19}$ Der Löwenanteil der Anträge verteilt sich auf die bevölkerungsreichen Bundesländer mit hohem Ausländeranteil, das heißt Nordrhein-Westfalen und Baden-Württemberg, sowie den Sonderfall Berlin, wo überdurchschnittlich viele ausreisepflichtige Ausländer residieren. Die Arbeitslast für die HFK im nach Fällen, Beratungen und Stattgaben etwas überraschend nächstfolgenden Bundesland, Schleswig-Holstein, ${ }^{20}$ betrug schon nur ein Sechstel derjenigen in Nordrhein-Westfalen. Die fehlenden Angaben zur Zahl der Anträge hat in Berlin und Sachsen mit der dezentralen Antragseinreichung über die Einzelmitglieder der HFK zu tun, welche darüber keine Statistik führen müssen, in Hamburg mit der Vorschaltung des Eingabeverfahrens, die einen direkten HFK-Antrag durch die Betroffenen verunmöglicht.

19 Ein Fall betrifft im Durchschnitt zwischen 2,5 und 3 Personen, da oft Anträge für ganze Familien gestellt werden.

20 Womöglich hat dies damit zu tun, dass es in Schleswig-Holstein schon vor der Einführung des $\S 23$ a eine Härtefallkommission gab, also Beratungsstellen eine gewisse Antragsroutine besaßen, und Anträge in diesem Bundesland zudem auch durch Dritte gestellt werden können. Zum anfänglichen Aufgabenspektrum der Vorläuferkommission und ihrer Arbeitsweise vgl. insbesondere den Jahresbericht der Härtefallkommission des Landes Schleswig-Holstein für 2001. 
Die Einbindung zivilgesellschaftlicher Akteure in die Härtefallkommissionen

Tabelle 2: Kumulierte HFK-Fall- und Entscheidungsstatistik 2005-2009

\begin{tabular}{|c|c|c|c|c|c|c|c|}
\hline Land & Anträge & $\begin{array}{c}\text { Anträge je } \\
1 \text { Mio. } \\
\text { Einwohner }\end{array}$ & $\begin{array}{l}\text { Beratungs- } \\
\text { quote }\end{array}$ & $\begin{array}{l}\text { Ersuchens- } \\
\text { quote }\end{array}$ & Stattgabequote & $\begin{array}{l}\text { Erfolg- } \\
\text { reiche } \\
\text { Fälle }\end{array}$ & $\begin{array}{l}\text { Erfolgs- } \\
\text { quote }\end{array}$ \\
\hline $\begin{array}{l}\text { Baden- } \\
\text { Württemberg }\end{array}$ & 1595 & 149,1 & 62,7 & 41,5 & 91,8 & 377 & 23,6 \\
\hline Bayern & 301 & 24,1 & 37,5 & 90,2 & 100 & 102 & 33,9 \\
\hline Berlin & I & I & 1 & 88,5 & 63,1 & 665 & 1 \\
\hline Brandenburg & 142 & 56,8 & 77,5 & 79,6 & 91,4 & 64 & 45,1 \\
\hline Bremen & 22 & 31,4 & 72,7 & 100 & 100 & 13 & 59,1 \\
\hline Hamburg & I & 1 & 1 & 49,4 & 100 & 79 & l \\
\hline Hessen & 339 & 55,6 & 54,9 & 61,8 & 93,1 & 95 & 28,1 \\
\hline $\begin{array}{l}\text { Mecklenburg- } \\
\text { Vorpommern }\end{array}$ & 113 & 66,5 & 40,7 & 56,5 & 69,2 & 18 & 15,9 \\
\hline Niedersachsen & 302 & 38,2 & 20,9 & 71,4 & 87,9 & 29 & 9,6 \\
\hline $\begin{array}{l}\text { Nordrhein- } \\
\text { Westfalen }\end{array}$ & 2656 & 148,4 & 76 & 22,8 & 95,8 & 367 & 13,8 \\
\hline Rheinland-Pfalz & 161 & 40,3 & 95,7 & 35,8 & 100 & 54 & 33,5 \\
\hline Saarland & 221 & 221 & 62,4 & 71,7 & 83,3 & 80 & 36,2 \\
\hline Sachsen & I & I & / & 71,1 & 71,9 & 46 & 1 \\
\hline Sachsen-Anhalt & 109 & 45,4 & 91,7 & 35 & 97,1 & 34 & 31,2 \\
\hline $\begin{array}{l}\text { Schleswig- } \\
\text { Holstein }\end{array}$ & 456 & 162,8 & 56,6 & 46,9 & 90,8 & 108 & 23,7 \\
\hline Thüringen & 230 & 100 & 83,5 & 69,4 & 83,3 & 100 & 43,5 \\
\hline
\end{tabular}

/ Keine Angabe bzw. Berechnung wegen fehlender Angaben nicht möglich. Eine Multiplikation der Antragszahlen mit den entsprechenden Quoten führt wegen nicht abschließend entschiedener Fälle nicht immer exakt zur angegebenen Zahl der erfolgreichen Fälle. Grau hinterlegt sind die für genauere qualitative Analysen ausgewählten Länder.

Quelle: Synopse der HFK-Strukturen und -Fallstatistiken von Andreas Schwantner (Amnesty International); teils eigene Berechnungen auf Basis dieser Daten sowie von Daten des Statistischen Bundesamtes.

Auch bei der Beratungsquote (dem Anteil der in der Kommission beratenen Anträge an allen Anträgen), der Ersuchensquote (dem Anteil der Ersuchen an den beratenen Anträgen) und der Stattgabequoten (dem Anteil der Ersuchen, in denen die oberste Landesbehörde kein Veto einlegte) zeigt sich eine große Variationsbreite. So wurde in Niedersachsen nur jeder Fünfte und in Hessen nur gut jeder zweite Antrag überhaupt beraten, in Nordrhein-Westfalen blieb dagegen trotz der hohen Antragszahlen 
nur ein Viertel ohne Beratung. Die Ersuchensquote streut ebenso breit, von etwas mehr als einem Fünftel in Nordrhein-Westfalen bzw. einem Drittel in RheinlandPfalz und Sachsen-Anhalt bis zu $100 \%$ in Bremen und angesichts der Fallzahlen vielleicht noch erstaunlicheren $88 \%$ in Berlin. Auf ein Veto haben manche Länder bislang komplett verzichtet, andere reduzieren die Stattgabequote damit auf bis knapp über 60\%, etwa Berlin. Manche der Extremwerte in den drei Quoten korrespondieren dabei auf auffällige Weise miteinander, so beispielsweise die sehr hohe Beratungsquote in Nordrhein-Westfalen mit einer sehr geringen Ersuchensquote und selten eingelegtem Veto.

Im Ergebnis sind in Niedersachsen nicht einmal zehn Prozent der Anträge erfolgreich, ${ }^{21}$ in Bremen dagegen fast $60 \%$. Die Länder mit hohen Antragszahlen liegen im Ergebnis trotz der größeren Variation in der Beratungs- und Ersuchenspraxis wieder relativ nah beieinander, besonders wenn man bedenkt, dass in NordrheinWestfalen im Rahmen des hier ja besonders intensiven Arbeitsebenenkontakts der HFK zu den Ausländerbehörden auch Empfehlungen in Bezug auf den eigentlich anspruchsvolleren $\S 104$ a AufenthG ausgesprochen wurden - diese ca. 40 Fälle fehlen dann in der Ersuchensstatistik - und durch die Delegation des Vetos an die Ausländerbehörden zuweilen eine größere Zeitverzögerung eintreten kann als andernorts, sodass dort am Stichtag der Erfassung der Daten für 2009 ungewöhnlich viele Fälle noch nicht entschieden waren. Dennoch kann festgehalten werden, dass der Zufall der Bundesländerzuweisung für Flüchtlinge zu einer Lotterie der Chancen auf einen Aufenthaltsstatus werden kann, was angesichts der existenziellen Bedrohungen in vielen Herkunftsländern durchaus als Skandalon betrachtet werden darf. $^{22}$

Experteninterviews wurden für den vorliegenden Artikel in Baden-Württemberg, Berlin, Hessen, Hamburg und Nordrhein-Westfalen durchgeführt. Teilnehmende Beobachtungen an je einer HFK-Sitzung kamen in Baden-Württemberg und Hessen

21 Nach einem Bericht der Hannoversche Allgemeine Zeitung vom 29.09.2010 kam es jüngst (nach einem ähnlichen Vorfall im Jahr 2007) erneut zu einer konflikthaften Zuspitzung innerhalb der niedersächsischen HFK. Ein der CDU angehörender ehemaliger Kommunalpolitiker zog sich unter Verweis auf die Entscheidungspraxis von HFK und Innenministerium aus der Kommission zurück, und Kirchenvertreter schlossen sich seiner Kritik an (Berger 2010: 5).

22 Diese Chancenungleichheit ähnelt im Übrigen derjenigen auf der europäischen Ebene im Dublin IISystem. Für Analysen sechs verschiedener nationalstaatlicher Entwicklungspfade inklusive des deutschen unter diesem System vgl. Menz 2009; zum (sehr eng begrenzten) Einfluss von NROs auf die Gestaltung der Asylverfahrensrichtlinie vgl. Seifer 2009. 
hinzu. ${ }^{23}$ Die ausgewählten fünf Länder decken mehr als drei Viertel der beratenen Anträge und mehr als zwei Drittel der Ersuchen sowie der erfolgreichen Fälle ab; die rein quantitative Relevanz dürfte also unstrittig sein. Eine hinreichende Variation in allen Quoten wäre bereits durch die „big three“ abgedeckt. Hessen wurde hinzugezogen, weil es hier die größte Reformaktivität gab, wodurch unter anderem eine recht stark vom Parteienwettbewerb geprägte HFK hinzukommt und die Arbeit in einer sehr großen Kommission beleuchtet werden kann; Hamburg ist als einziger Fall einer rein parlamentarisch besetzten HFK von besonderem Interesse.

\section{b) Qualitative Analyse der Entscheidungspraxis}

In allen näher untersuchten Fällen festzustellen ist ein hoher Grad an Identifikation der Mitglieder mit ihrer Härtefallkommission und die Neigung, die Regelungen und Verfahrensweisen im eigenen Bundesland für besser als in den anderen zu halten. Auch das Arbeitsklima wurde in allen fünf Ländern, zu denen Experteninterviews geführt wurden, gelobt. Die Tatsache, mehreren tausend Menschen eine Bleibeperspektive eröffnet zu haben, wurde durchweg als erfreulich hervorgehoben.

Der für den deutschen Föderalismus typische kritische Blick aus den übrigen Landeshauptstädten nach Berlin findet sich auch im Kontext der HFKs: Mehrfach wurde in den Experteninterviews von staatlichen Vertretern vorsichtige Verwunderung bis offensives Unverständnis über die hohe Zahl von Empfehlungen, die die Berliner HFK ausspricht, geäußert, und diese mit der zumindest vermeintlich problematischeren dortigen Population in Kontrast gesetzt. Eine weitere Ähnlichkeit $\mathrm{zu}$ anderen Bundesländervergleichen scheint in den andernorts zu hörenden Einschätzungen über den zumindest von sich selbst als solchen angesehenen Klassenprimus Baden-Württemberg auf. In Abgrenzung zu diesem wird etwa von staatlichen wie NRO-Vertretern in der nordrhein-westfälischen HFK die große rechtliche Korrektheit des eigenen Vorgehens in Bezug auf die Ausschlussgründe betont. „Die HFK soll sich nicht an die Stelle des Gesetzgebers stellen“ (so ein staatliches Mitglied).

23 Andernorts wurden entsprechende Anfragen unter Verweis auf die sensible Natur der Fallakten, die prinzipielle Nichtöffentlichkeit der Sitzungen und die Verschwiegenheitspflicht der Mitglieder abgelehnt. Umso mehr ist der Autor den Verantwortlichen und HFK-Mitgliedern in Baden-Württemberg und Hessen zu Dank für ihre Aufgeschlossenheit verpflichtet und um einen verantwortungsbewussten Umgang mit den dort gewonnenen Einblicken bemüht. 
Gewisse Unterschiede im Arbeitsklima ergeben sich aus den unterschiedlich großen Kontinuitäten in der Besetzung und den jeweiligen Gruppengrößen. ${ }^{24}$ So kennen sich in Baden-Württemberg die neun HFK-Mitglieder besonders lange und eng, man nimmt treffsicher vermutete Positionen anderer Mitglieder in Debattenbeiträgen bereits vorweg, geht im Anschluss gemeinsam essen und hält eine Weihnachtsfeier ab. In Hessen dagegen fällt unter den 23 Mitgliedern schon anhand der (nicht vorgegebenen) Sitzordnung eine klare Lagerbildung zwischen staatlichen und insbesondere NRO-Vertretern auf, aber auch innerhalb der großen Gruppe der Ersteren - hier sitzen etwa die kommunalen Vertreter und die Parlamentarier ${ }^{25}$ jeweils beieinander. Zudem werden hier in der Praxis Fälle ausschließlich von der NRO-Seite und Oppositionsvertretern eingebracht, und das Ganze hat etwas PolitikbetriebsGeschäftsmäßigeres.

Elf der sechzehn HFKs veröffentlich(t)en mittlerweile bzw. zwischenzeitlich Tätigkeitsberichte. Der baden-württembergische enthält typisierende Darstellungen von behandelten Fällen, welche die Bandbreite der humanitären Erwägungen in den HFKs illustrieren und deshalb im Folgenden ausführlich zitiert seien.

24 Empirisch mehr oder weniger robusten, aber nahezu einhelligen Thesen aus soziologischen und psychologischen Arbeiten zu Gruppenentscheidungen zufolge sind hohe einstellige Zahlen für die Interaktion und Kohärenz der Gruppe ideal (vgl. etwa Ephross/Vassill 1998: 137; Mills 1967: 64). Indes kann Kohärenz durch die Institutionalisierung einer größeren Gruppe auch bewusst begrenzt werden, kann doch ,[n]iedrige Gruppenkohärenz [...] den Vorteil haben, daß die Mitglieder nicht zu stark in den Gruppenprozeß einbezogen sind. Sie erliegen nicht oder weniger dem Konformitätsdruck, sie internalisieren nicht voreilig die impliziten Normen der Gruppe und sie sind der Gruppenloyalität weniger verpflichtet. Sie haben dann in höherem Maße die Möglichkeit, sich eine unabhängige Meinung zu bilden und diese in der Gruppe zu vertreten. Solche Meinungen können für die Gruppe wertvoller sein als Aussagen, die bereits stark von der Gruppe und ihrem Normensystem beeinflußt sind.“ (Sader 1976: 140). Bei etwas mehr Muße lohnt auch ein Blick in das Kapitel über „Die quantitative Bestimmtheit der Gruppe“ bei Simmel 1908.

25 Deren vermeintliche Sonderrolle wurde im hochkontroversen Anhörungsverfahren zur jüngsten Gesetzesnovelle - ein Blick ins Ausschussprotokoll ist höchst empfehlenswert - aber wohl überschätzt, zumal es offenkundig auch innerhalb dieser Gruppe ganz unterschiedliche Positionierungen und Rollenauffassungen gibt. 


\section{Einschub 1: Typen von Härtefällen und ihre Behandlung}

Eine Familie mit langjährigem Aufenthalt in der Bundesrepublik hat sechs in Deutschland geborene Kinder, die von deren Grund- und Hauptschullehrern insgesamt ordentlich beurteilt wurden. Die wirtschaftliche und soziale Integration der Eltern, welche zwischendurch das Bundesgebiet kurzfristig auch wieder verlassen hatten, entsprach aber in keiner Weise den sonst üblichen Anforderungen der HFK. Gleichwohl richtete die HFK im Hinblick auf die Entwicklung der sechs hier geborenen Kinder trotz der durch den Aufenthalt der Familie bereits eingetretenen und noch zu erwartenden Belastungen für die Steuerzahler ein Ersuchen an den Innenminister, dem auch entsprochen wurde. [...]

Nicht erfolgreich war der Antrag eines Mannes, dessen Integrationsbemühungen trotz langjährigen Aufenthalts in Deutschland sowohl in wirtschaftlicher als auch sozialer Hinsicht nur wenig überzeugen konnten. Er kam zudem verschiedentlich mit dem Gesetz in Konflikt. [...]

Schon nach einem relativ kurzen Aufenthalt war hingegen der Antrag eines gerade volljährig gewordenen jungen Mannes erfolgreich. Er hat eine Lehre begonnen und wird vom Ausbildungsbetrieb gelobt. Auch eine gelungene soziale Integration wurde von verschiedenen Bürgern und Vereinen nachdrücklich bestätigt. Eine Rückkehr in seine Heimat wäre zwar kaum eine unzumutbare Härte, aber die Kommission sah dennoch keinen triftigen Grund, einen so gut integrierten und erfolgversprechenden jungen Mann aus seinem sozialen Umfeld herauszureißen und hat deshalb ein Ersuchen an den Innenminister gestellt, dem auch entsprochen wurde.

Weniger befriedigend wurde der Fall einer seit 19 Jahren hier lebenden Frau gelöst, obgleich sie seit über sieben Jahren eine ungekündigte Stelle für einfachere Arbeiten hatte und auch sonst sozial integriert erschien. Sie hatte jedoch schon zu Beginn ihres Aufenthalts mit Hilfe einer gefälschten Urkunde über ihre Identität getäuscht und diese Urkunde in den folgenden Jahren immer wieder bei Behörden vorgelegt. Das Gericht sah darin mehrere mittelbare Falschbeurkundungen als jeweils selbständige Taten und verhängte deshalb eine längere auf Bewährung ausgesetzte Gesamtfreiheitsstrafe. Die Kommission gewichtete den langen Aufenthalt und die Integration der Frau etwas höher als die zweifellos erhebliche Straftat und stellte ein Härtefallersuchen, dem der Innenminister der hohen Strafe wegen jedoch nicht entsprechen konnte.

Quelle: Härtefallkommission beim Innenministerium Baden-Württemberg (2009: 8 f.).

Nahezu allgemeiner Tenor nicht nur bei den nichtstaatlichen Vertretern ist des Weiteren, dass die wirtschaftliche Dimension der Integration von den Landesgesetzbzw. -verordnungsgebern überbetont worden sei und leider zu viele ansonsten sehr gut integrierte Antragsteller am entsprechenden Regelausschlussgrund scheiterten. ${ }^{26}$ Dies gelte zumal für Familien mit vielen Kindern, die auch bei Vollzeiterwerbstätigkeit oft auf ergänzende Sozialleistungen angewiesen seien. Mehrere Mitglieder der baden-württembergischen HFK gaben indes an, diese wende den betreffenden Regelausschlussgrund schlicht so nicht an. Da aus Landesregierung und Landtag praktisch keine Kritik an der HFK-Arbeit komme, sondern tendenziell die Botschaft, dass man über diese sehr froh sei (Schlagzeilen über die Abschiebung im Ort beliebter Mitglieder von Kirchengemeinde oder Fußballverein, deren Kinder schulisch erfolgreich sind, mag auch so mancher innenpolitische Hardliner im ei-

26 In diesem Zusammenhang sei auf die Kritik am dahinter stehenden Modell von ,social citizenship“ etwa bei Apitzsch 2003 zumindest verwiesen. 
genen Wahlkreis nicht lesen), vermerke man den Sozialleistungsbezug durchaus negativ, gewichte aber andere positive Faktoren höher. Aus anderen Ländern hört man dazu zum einen, dass es in Baden-Württemberg ohnehin viel leichter sei, Arbeit zu finden, und zum anderen, dass der Vetospieler oberste Landesbehörde sich anderswo darauf nicht in dem Maße einließe. Eine nicht unbeträchtliche Binnenvariation sei in dieser Frage anfangs in Nordrhein-Westfalen zu konstatieren gewesen, wo das Veto ja an die kommunalen Ausländerbehörden delegiert ist. Hier muss sich der Leiter der Ausländerbehörde vor dem Kämmerer für Entscheidungen mit Kostenfolgen rechtfertigen, und nicht wenige Kommunen stehen zudem unter Haushaltsaufsicht, was solche Fragen potenziell noch virulenter macht. Inzwischen hat sich die Stattgabequote in Nordrhein-Westfalen aber trotz des zersplitterten Vetospielers auf $100 \%$ eingespielt. ${ }^{27}$ In Hessen haben die kommunalen Kostenträger die Möglichkeit, einem wegen nur partieller oder ganz fehlender Lebensunterhaltssicherung eigentlich abzulehnenden Antrag durch ihr Einvernehmen zur weiteren Leistungserbringung zum Erfolg zu verhelfen. ${ }^{28}$

Obgleich seit der Einrichtung der HFKs nach $\S 23$ a AufenthG erst wenige Jahre ins Land (bzw. die Länder) gezogen sind, gibt es einige erwähnenswerte Aspekte der Entwicklung über die Zeit. So konnte die HFK in Baden-Württemberg ihre Stattgabequote von anfänglich ca. 80\% auf 100\% im Jahr 2009 steigern, was nicht zuletzt an der personellen Konstellation im Innenministerium einerseits und dem Engagement, Standing und Selbstverständnis des Kommissionsvorsitzenden andererseits lag, welches sich auch auf die Kommission übertrug. Diese sieht sich auf staatlicher wie auf zivilgesellschaftlicher Seite als Entscheidungsinstanz - ,wir sind eine Autorität", so ein der letzteren Gruppe angehörendes Mitglied der Kommission im Expertengespräch. Inwiefern solche personalen Effekte sich auf die Institution als solche übertragen, Neubesetzungen in Ministerium und HFK also überdauern können, muss indes cum grano salis betrachtet werden.

27 Manche Ausländeramtsleiter reagierten auf die Vetoübertragung an diese anfangs mit ,echtem Entsetzen“ (ein staatliches Mitglied der NRW-HFK) darüber, dass nach dem vor einem HFKVerfahren ja üblichen Gang durch viele Instanzen nun der schwarze Peter wieder bei den Ausländerämtern liegen sollte; inzwischen habe sich aber eine hohe Akzeptanz der Lösung ergeben, obwohl die tatsächliche Ausübung des Vetos auf kommunaler Ebene aufgrund der politischen Abhängigkeiten schwieriger sei als auf Landesebene.

28 Entsprechende Verpflichtungserklärungen von Angehörigen erwiesen sich in verschiedenen Ländern immer wieder als nicht dauerhaft belastbar (vgl. hierzu auch Wais 2010: 256), oft wegen unzureichendem Einkommen der Angehörigen, zuweilen auch wegen familiärer Differenzen. Aus dem hessischen Tätigkeitsbericht für 2010 geht hervor, dass ,,das zwingende Erfordernis [...] eines ausreichenden Krankenversicherungsschutzes besonders schwer zu überwinden ist [, i]nsbesondere [für] Alte, Kranke und traumatisierte Ausländerinnen und Ausländer.“ (Geschäftsstelle der Härtefallkommission in Hessen 2011: 16) 
Rückläufig in der Zeit ist die zuvor extrem hohe Beratungsquote in NordrheinWestfalen. Dies dürfte weniger an Ermüdungserscheinungen in der HFK liegen als an der (auch anderswo zu konstatierenden) Tendenz, dass der Pool der vergleichsweise aussichtsreichen Antragsteller etwas kleiner und der Anteil völlig aussichtsloser Fälle größer geworden ist. Die Fallzahlen sind in allen Ländern seit 2007 geringer als zuvor, da es einerseits einen großen angestauten Bedarf gab und andererseits verschiedene Altfallregelungen greifen oder griffen. Diese ermöglichen es den Ausländerbehörden bei einem Aufenthalt von acht oder mehr Jahren und der Erfüllung einiger weiterer Bedingungen, geduldeten Ausländern auch ohne Rekurs auf die humanitäre Situation und damit ohne Anrufung der HFK eine Aufenthaltserlaubnis zu erteilen. (Zuvor waren die HFKs de facto auch zum Überlaufbecken für diese Altfälle geworden und sind erst seither auf ihre eigentliche Funktion beschränkt.) So haben sich der Tagungsrhythmus, die Sitzungsdauer und der Vorbereitungsaufwand für die HFK-Mitglieder deutlich reduziert. In Baden-Württemberg etwa tagte man anfangs 14-tägig bei ca. 25-30 Fällen pro Sitzung - angesichts der oft 50 oder mehr Seiten umfassenden Anträge je Fall ein wirklich beachtlicher Zeitaufwand gerade für die ehrenamtlichen Mitglieder ${ }^{29}-$, kommt inzwischen aber nur noch alle 6-8 Wochen für 10-12 Fälle zusammen. Zu bedenken ist allerdings, dass nach dem Eindruck zahlreicher Gesprächspartner unter den derzeit gestellten Anträgen der Anteil komplexer Fälle weit höher ist als zu Beginn, in denen positive und negative (Integrations-)Faktoren gegeneinander abzuwägen und in Familien oft asymmetrisch verteilt sind. Dies erschwere und verlängere tendenziell die Entscheidungsfindung.

Viele Gesprächspartner berichteten, und die teilnehmenden Beobachtungen lieferten weitere Belege dafür, dass es in solchen Fällen oft keine klaren Muster im Argumentations- und Abstimmungsverhalten der HFK-Mitglieder nach von ihnen vertretener Stelle gebe, sondern dass persönliche Sichtweisen und Wertungen mehr Gewicht erhielten. ${ }^{30}$ So könne ein Kirchenvertreter bei Antragstellern mit Vorstrafen auch einmal härter erscheinen als ein Vertreter eines kommunalen Verbandes oder ein Wirtschaftsvertreter bei Identitätsverschleierung großzügiger als ein Inte-

29 Explizit festgehalten werden sollte, dass gerade auch auf der staatlichen Seite ein Großteil der notwendigen Vorbereitungszeit ehrenamtlich in der Freizeit geleistet wird, nicht nur bei den mancherorts besonders gerne auf die staatlichen Plätze in der HFK berufenen Pensionären.

30 Auch dies ist ein Beispiel für die Nützlichkeit der Differenzierung in ,[i]nnere und äußere Determinanten des Verhaltens von Asylrekursorganen“ (Holzer/Schneider 2002: 135). 
grationsbeauftragter. ${ }^{31}$ Holzschnittartigen Hypothesen widerspricht es zudem, wenn ein Ausländeramtsleiter darüber konsterniert ist, ,,wie man zum Beispiel das tiefe, tiefe Elend ignorieren kann, in das Roma im Kosovo fallen" und hier über die HFK froh ist, während von Seiten einer Frauenrechtsorganisation einem homosexuellen Araber unterstellt wird, sein Outing sei solange nicht glaubhaft, bis er sich öffentlich für Homosexuellenrechte engagiere. Das Geschlecht der HFK-Mitglieder wurde in Interviews indes als eine zuweilen entscheidende Determinante des Abstimmungsverhaltens über bestimmte Fälle benannt. ${ }^{32}$

Sowohl aus der baden-württembergischen als auch aus der nordrhein-westfälischen HFK wird nichtsdestoweniger von einem Trend zu mehr einstimmigen Entscheidungen berichtet. Das liege zum einen daran, dass NRO-Vertreter in aussichtslosen Fällen auch einmal mit nein stimmten, um an Glaubwürdigkeit im Gremium zu gewinnen und dadurch in strittigen Fällen auch eher einmal mit ihren Argumenten überzeugen zu können. Nahezu durchweg wird von interviewten staatlichen Vertretern hervorgehoben, dass die Prozess- und Entscheidungsqualität der HFKs von der NRO-Inklusion profitiere: „Ohne die NROs könnten die Entscheidungen so nie getroffen werden. Die ganze Erfahrung, die die NROs mitbringen, ist nicht zu ersetzen" (ein HFK-Vorsitzender). Kritischere Haltungen gibt es allerdings zur Hinzuziehung von Flüchtlingsräten und ähnlichen schon vom Selbstverständnis her prinzipiell flüchtlingsfreundlichen Organisationen, denen oft unterstellt wird, sie könnten bei Abstimmungen gar nicht nein sagen - was empirisch so ausnahmslos nicht zutrifft. Manche wollten oder könnten sich auch auf das Ausländerrecht nicht einlassen, zu dem $\S 23$ a und die Härtefallverordnungen eben auch gehörten (so ein staatliches HFK-Mitglied). ${ }^{33}$ Andererseits wird von demselben Gesprächspartner anerkennend auf eine sehr hohe Fachkompetenz verwiesen, die bei manchem Vertreter der staatlichen Seite fehle.

Nicht zu vernachlässigen ist des Weiteren der Zugewinn an Erfahrung und Routine in der HFK-Arbeit. So sagte ein HFK-Mitglied im Expertengespräch ganz offen,

31 Deshalb kann es für die Aussichten eines Antragstellers durchaus auch einmal bedeutsam sein, wenn nicht ein in die HFK gewissermaßen hineinsozialisiertes HFK-Mitglied an einer Sitzung teilnimmt, sondern sein selten oder gar erstmals teilnehmender Stellvertreter. Der Vorsitzende der baden-württembergischen HFK, Dr. Edgar Wais, sprach in diesem Zusammenhang in der Anhörung des Innenausschusses des hessischen Landtages am 24.9.2009 gar von der Möglichkeit von „Zufallsmehrheiten“.

32 Zur allgemeinen Lage von weiblichen Flüchtlingen und Asylsuchenden vgl. im Übrigen Hunt 2008.

33 Auch auf den Ausländerämtern erlebe man viel Empirisches, was die Flüchtlingslobby nicht sehen wolle, z. B. Identitätsverschleierungen nicht nur aus Not, sondern auch im Kontext krimineller Clans, Migranten, die über Jahrzehnte keine ernsthaften Versuche zur Arbeitsaufnahme oder zum Spracherwerb unternähmen, oder 12-14-jährige Mädchen, die von Bildung ferngehalten und im Haushalt ausgebeutet würden. 
dass er als Antragsteller lieber heute als vor vier Jahren von der Kommission behandelt würde, da man inzwischen professioneller, differenzierter und damit gerechter auf die Fälle einzugehen gelernt habe. „Jedes Mitglied hat sich inzwischen mit 1650 persönlichen Schicksalen befasst, und das hat verändernden Charakter“ (Zitat aus dem Interview mit einem Mitglied der baden-württembergischen HFK). $\mathrm{Zu}$ einer gewissen Annäherung der Entscheidungspraxis bei bestimmten Falltypen haben nach Aussagen verschiedener Interviewpartner auch jährliche Treffen der HFK-Vorsitzenden (und je eines weiteren Mitglieds) beim Bundesamt für Migration und Flüchtlinge beigetragen. ${ }^{34}$

\section{c) Quantifizierende Analyse der Entscheidungspraxis}

Eine quantifizierende Analyse der Entscheidungsstatistiken bestätigt grosso modo die in den Expertengesprächen oft geäußerte Auffassung, dass persönliche Haltungen der HFK-Mitglieder und die Vielfalt der behandelten Einzelschicksale eine systematische Musterausbildung weitgehend verhindern. Einige der in Tabelle 3 aufgeführten bivariaten Korrelationen sind dennoch erwähnenswert: So wird anhand der ersten beiden Zeilen deutlich, dass eine größere Beteiligung der Zivilgesellschaft jedenfalls eindeutig keine positiven Auswirkungen auf die Höhe der Ersuchens-, Stattgabe- und Erfolgsquoten hat. (Sowohl die Prozess- als auch die Ergebnisqualität - diese Unterscheidung entleihe ich aus dem Bundesländervergleich der Strafvollzugsprivatisierung bei Töller/Dittrich 2010: 23 - kann selbstverständlich nichtsdestoweniger auch bei nicht nachweisbarem Einfluss auf diese drei Quoten erhöht worden sein.)

34 Nach Kenntnis des Verfassers treffen sich auch die kirchlichen HFK-Mitglieder und von der Partei Bündnis 90/Die Grünen nominierte HFK-Mitglieder regelmäßig zum länderübergreifenden Austausch (diese Angabe geschieht ohne Anspruch auf Vollständigkeit). Aus beiden Kreisen wird von einer qualitäts- und homogenitätssteigernden Wirkung dieser Treffen auf die je länderspezifische HFK-Mitarbeit berichtet, insbesondere im Hinblick auf die Sensibilisierung für bestimmte Falltypen und Strategien im Umgang mit diesen. Bei den kirchlichen Treffen wurden neben Handlungsbedarf auf Seiten der Politik, etwa bezüglich des Regelausschlussgrundes Sozialleistungsbezug, auch Desiderata zu den Arbeitsbedingungen der HFK-Mitglieder identifiziert. So wurde gerade in Ländern mit hohen Fallzahlen eine entsprechende Ausstattung und juristische Beratung der HFKMitglieder durch die Kirchen angemahnt. 
Tabelle 3: Quantifizierende Analysen der Entscheidungsstatistik I: Korrelationen

\begin{tabular}{|c|c|c|c|c|}
\hline & $\begin{array}{l}\text { Beratungs- } \\
\text { quote }\end{array}$ & $\begin{array}{c}\text { Ersuchens- } \\
\text { quote }\end{array}$ & $\begin{array}{l}\text { Stattgabe- } \\
\text { quote }\end{array}$ & $\begin{array}{c}\text { Erfolgs- } \\
\text { quote }\end{array}$ \\
\hline Anzahl zivilgesellschaftlicher Mitglieder & $-0,08$ & $-0,002$ & $-0,26$ & $-0,28$ \\
\hline Beschlussfähige Mehrheit nichtstaatliche Seite & 0,05 & $-0,21$ & $-0,23$ & $-0,38$ \\
\hline Flüchtlingsräte/Pro Asyl vertreten & 0,46 & $-0,07$ & $-0,27$ & 0,17 \\
\hline Ausländeranteil 2008 & $-0,16$ & 0,11 & 0,22 & 0,04 \\
\hline $\begin{array}{l}\text { Ausländer abhängig von öffentlichen Leistungen } \\
2005\end{array}$ & 0,25 & 0,12 & $-0,51 * *$ & 0,17 \\
\hline Ost-West-Dummy & 0,29 & 0,09 & $-0,52 * *$ & 0,17 \\
\hline BIP pro Kopf 2009 & $-0,20$ & 0,12 & $0,52 * *$ & 0,28 \\
\hline Einrichtende Regierung bürgerlich & $-0,25$ & $-0,03$ & 0,3 & $-0,09$ \\
\hline Einrichtende Regierung links & $-0,16$ & $-0,22$ & $-0,46^{*}$ & $-0,53 *$ \\
\hline Einrichtende Regierung große Koalition & 0,22 & $0,48^{*}$ & $-0,04$ & $0,69 * * *$ \\
\hline Kumulierte Unions-Kabinettssitzanteile & $-0,37$ & $-0,05$ & 0,18 & $-0,22$ \\
\hline Nichtbefassungsgründe & $-0,43$ & $-0,22$ & 0,03 & $-0,55^{* *}$ \\
\hline SPD-Innenminister $^{\mathrm{a}}$ & 0,22 & $-0,30$ & $-0,28$ & $-0,22$ \\
\hline $\mathrm{N}$ & 13 & 16 & 16 & 13 \\
\hline
\end{tabular}

* steht für ein Signifikanzniveau von 90\%, ** für 95\% und *** für 99\%.

a Ohne den Fall Nordrhein-Westfalen, wo das Veto ja an die Ausländerbehörden delegiert ist, liegt der Wert für die Stattgabequote bei -0,25 und für die Erfolgsquote bei -0,32.

Untersucht wurde die kumulierte Statistik der Jahre 2005 bis 2009. Die niedrigere Fallzahl bei der Beratungs- und Erfolgsquote ergibt sich aus den Lücken in der Erfassung der eingegangenen Anträge (siehe Tabelle 2). Die Daten zur Abhängigkeit der Ausländer von öffentlichen Leistungen lagen für die neuen Länder einerseits (38\%) und Bremen und Niedersachsen andererseits (19\%) nur als Durchschnitt vor, sodass hier die Trennschärfe eingeschränkt ist. Im Fall von Bremen und Hessen, wo es Reformen der HFK-Besetzung gab, wurde bei den erklärenden Variablen ggf. jeweils die Konstellation berücksichtigt, unter der die (große) Mehrzahl der Fälle bearbeitet wurde. Selbiges gilt für die SPD-Innenminister-Variable.

Die Präsenz von flüchtlingsfreundlichen NROs in den Härtefallkommissionen führt dazu, dass deutlich mehr Fälle beraten werden; letztlich hat sie auf die Erfolgsquote allerdings nur einen schwach positiven Einfluss. ${ }^{35}$ Der Anteil an Ausländern, die von öffentlichen Leistungen abhängig sind, ist mit der Stattgabequote recht hoch

35 Nimmt man das besonders beratungsfreudige Nordrhein-Westfalen heraus, springt die Korrelation der entsprechenden Variable mit der Erfolgsquote auf 0,28 und diejenige mit der Ersuchensquote wird zur schwarzen Null. 
negativ korreliert, was darauf hindeutet, dass es den Innenministern in den entsprechenden Ländern besonders schwer fällt, ihr Vetorecht zurückhaltend auszuüben, weil dies von der Opposition als Laxheit gegeißelt werden könnte. Allerdings ist dieser Indikator mit einer Ost-West-Dummy-Variable (Kodierung: Ostländer 1, Westländer 0, Berlin 0,5) extrem hoch interkorreliert, sodass potenziell auch andere für die neuen Länder typische Konstellationen, etwa die weitaus geringere Kirchenbindung, verantwortlich sein könnten. Die am BIP pro Kopf bemessene Wirtschaftskraft eines Landes scheint tatsächlich sowohl die Stattgabe- als auch die Erfolgsquote (nicht aber die Beratungsquote) positiv zu beeinflussen, wie von manchen Gesprächspartnern gerade in wirtschaftsschwächeren Ländern (nicht ganz ohne Neid) vermutet wurde. Insbesondere die wirtschaftliche Integration der Antragsteller dürfte hier tendenziell erfolgreicher verlaufen sein. Das Erbe der politischen Färbung derjenigen Regierung, die die jeweilige HFK dereinst eingerichtet hat, kommt insbesondere in unterdurchschnittlichen Stattgabe- und Erfolgsquoten der von linken Regierungen eingerichteten HFKs sowie in überdurchschnittlich hohen Ersuchens- und Erfolgsquoten großkoalitionär begründeter Kommissionen zum Tragen. Dieser letzte Befund ist jedoch in seiner Bedeutung insofern einzuschränken, als der Erfolgsquotenbetrachtung mangels Angaben zu den Antragszahlen aus Sachsen lediglich die beiden vergleichsweise fallarmen großkoalitionären HFKs aus Bremen und Brandenburg zugrunde liegen. Bürgerliche Regierungen sowie Unionskabinettssitzanteile zeitigen hingegen zwei kleinere Effekte, einen positiven auf die Stattgabe- und einen negativen auf die Erfolgsquote. Letzteren teilen sie mit den SPD-Innenministern, welche womöglich stärker unter dem Druck stehen, sich als „harte Hunde“ beweisen zu müssen. ${ }^{36}$ Die schiere Anzahl der Nichtbefassungs- und Regelausschlussgründe - anhand der vorherigen Ausführungen dürfte deutlich sein, dass dies nur ein grober Indikator ist - steht in einem klaren negativen Zusammenhang mit der Beratungs- und der Erfolgsquote.

36 Hier fallen gewisse Parallelen zur von Schmidt (1980: 113) untersuchten Umsetzung des Radikalenerlasses in den Bundesländern auf. 
Tabelle 4: Quantifizierende Analysen der Entscheidungsstatistik II: Regressionen

\begin{tabular}{|l|c|c|c|c|}
\hline & $\begin{array}{c}\text { Beratungs- } \\
\text { quote }\end{array}$ & $\begin{array}{c}\text { Ersuchens- } \\
\text { quote }\end{array}$ & $\begin{array}{c}\text { Stattgabe- } \\
\text { quote }\end{array}$ & $\begin{array}{c}\text { Erfolgs- } \\
\text { quote }\end{array}$ \\
\hline Beschlussfähige Mehrheit nichtstaatliche Seite & & $-3,23$ & & \\
\hline Flüchtlingsräte/Pro Asyl vertreten & 15,95 & & & \\
\hline Ausländer abhängig von öffentlichen Leistungen 2005 & & & $-0,35$ & \\
\hline BIP pro Kopf 2009 & & & 0,0003 & 0,0002 \\
\hline Einrichtende Regierung links & & & $-9,76$ & \\
\hline Einrichtende Regierung große Koalition & & 23,37 & & $23,92^{* * *}$ \\
\hline Nichtbefassungsgründe & $-2,28$ & & & $-2,26 * *$ \\
\hline SPD-Innenminister & & $-6,36$ & & \\
\hline Konstante & 78,8 & $60,19 * * *$ & $90,08^{* * *}$ & $44,92^{* * *}$ \\
\hline $\mathrm{N}$ & 13 & 16 & 16 & 13 \\
\hline $\mathrm{R}^{2}$ & 0,31 & 0,26 & $0,45 *$ & 0,77 \\
\hline $\mathrm{R}_{\text {korr }}^{2}$ & 0,17 & 0,07 & 0,32 & 0,69 \\
\hline
\end{tabular}

* steht für ein Signifikanzniveau von $90 \%$, ** für $95 \%$ und *** für $99 \%$.

Tabelle 4 zeigt Best-Fit OLS-Regressionsmodelle zu den vier schon bislang untersuchten Quoten. Aus diesen (und dem Identifikationsprozess, der zu ihrer Auswahl führte) ist wiederum zu schließen, dass die systematische Variation und damit die Erklärungskraft der Modelle wie der einzelnen unabhängigen Variablen (und ihre Signifikanz ${ }^{37}$ ziemlich überschaubar sind, wenngleich mit einer gewissen Variationsbreite. Nur zwei unabhängige Variablen, die Präsenz von flüchtlingsfreundlichen NROs sowie die Anzahl der Nichtbefassungs- und Regelausschlussgründe, leisten einen nennenswerten Beitrag zur nur sehr begrenzt gelingenden Erklärung der Beratungsquote. Im Hinblick auf die Ersuchensquote und ihre quantifizierende Analyse ist der Autor versucht, von einer Black Box HFK zu sprechen - die innere Dynamik der HFKs, die sich in dieser Größe besonders manifestiert, folgt kaum äußeren strukturellen Determinanten, wie auch oben mittels der qualitativen Ana-

37 Zur Problematik von Signifikanztests bei Vollerhebungen sowie einschlägigen Begründungsfiguren und Interpretationsfragen vgl. Wolf 2006: $156 \mathrm{ff}$. 
lysen gezeigt werden konnte. ${ }^{38}$ Etwas besser erklärt werden kann im Zusammenspiel von sozioökonomischen und parteipolitischen Determinanten die Stattgabequote. $\mathrm{Zu}$ diesem Modell ist anzumerken, dass in alternativen Modellen der Effekt von SPD-Innenministern nur dann negativ ausfällt, wenn weder der Anteil der von öffentlichen Ausgaben abhängigen Ausländer noch der Ost-West-Dummy und das BIP pro Kopf inkludiert werden. Allerdings ist hier bezüglich der Relation zwischen bivariater und multivariater Analyse zu beachten, dass diese Variablen nicht auf derselben Ebene ansetzen: Der Innenminister bleibt auch bei Berücksichtigung anderer Größen der Veto-Träger, das heißt er mag von den Verhältnissen getrieben sein, aber diese entscheiden nicht für ihn. Die Erfolgsquote lässt sich vergleichsweise gut mithilfe der einrichtenden Regierung und der Zahl der Nichtbefassungsund Regelausschlussgründe erklären, auch wenn dieses ebenfalls schlanke Modell sicher im Kontext betrachtet werden muss und nur ein Puzzleteil der hier insgesamt vorgelegten Analysen sein kann. Auf die Erfolgsquote ist in ebenfalls nicht abgedruckten (comparatively worse-fit-)Modellen der Effekt einrichtender Linksregierungen (fast signifikant) negativ, allerdings bei dann geringerem Determinationsniveau. Zudem bleibt in alternativen Modellen zur Erfolgsquote der negative Effekt einer nichtstaatlichen Mehrheit auch dann bestehen, wenn Nordrhein-Westfalen ausgeschlossen wird.

\section{d) Identitäts- und Legitimationskonflikte auf Seiten der Nichtregierungsor- ganisationen}

Machtstrukturen könn(t)en indes nicht nur Auswirkungen auf die Schicksale der Flüchtlinge haben, sondern - wenn auch auf ungleich weniger existenzielle Weise ${ }^{39}$ - auch auf die Gestaltungsmöglichkeiten und das Selbstverständnis Sozialer Arbeit (vgl. Muy/Sulimma i.E.). Auf Seiten der Nichtregierungsorganisationen sind davon die Folgen der Mitwirkung in den HFKs für das (insbesondere sozialarbeiterischkaritative) Selbstverständnis der betreffenden Organisationen sowie die Glaubwürdigkeit gegenüber Mitgliedern und Klientel berührt. So wurde innerhalb der meisten

38 Erwähnenswert bleibt vielleicht zudem, dass der negative Effekt der NRO-Mehrheit auch in der multivariaten Überprüfung bestehen bleibt, wenn auch mit (hier nicht abgedrucktem) eher kleinem standardisierten partiellen Regressionskoeffizienten. Nimmt man allerdings das besonders beratungsfreudige Nordrhein-Westfalen aus dem Sample, kippt der Effekt ins Positive. (Der SPDInnenministereffekt bleibt dann nicht nur negativ, sondern wird signifikant. Für diese Variable ist dies genau genommen auch der korrekte Modellzuschnitt, da das Veto in Nordrhein-Westfalen an die Ausländerbehörden delegiert ist.)

39 Für eine Agamben'sche Perspektive auf Flucht und Asyl vgl. Puumala/Pehkonen 2010 und Buckel/ Wissel 2010, für eine Diskussion der Einwände Derridas gegen Kants Darlegungen zur Gastfreundschaft Brown 2010. 
NROs die ursprüngliche Entscheidung zur Mitwirkung durchaus auch kritisch diskutiert, und insbesondere in den Flüchtlingsräten flammen hierzu auch weiterhin teils kontroverse Debatten auf. Bei den interviewten NRO-Vertretern, die sich in ihren Entsendeorganisationen und der weiteren NRO-Community jeweils also durchaus für ihr Engagement rechtfertigen müssen, herrscht jedoch durchweg die Position vor, es sei richtig, weiter in den HFKs mitzuarbeiten, obgleich diese von Kritikern als Feigenblatt eines restriktiven humanitären Aufenthaltsrechts gebrandmarkt werden könnten. Aus der Sicht eines interviewten HFK-Vorsitzenden liegt einer der Vorteile der HFK-Teilnahme für die NROs im „Einfluss auf die ungeschriebenen Grundsätze im Gremium“ - wenngleich die HFK-internen Diskussionsprozesse sicher nicht jedem Flüchtlingsberater außerhalb der Kommissionen einleuchteten.

Ein NRO-Vertreter bemerkte allerdings (selbst-)kritisch, in den Ländern, in denen gerade auch die Flüchtlingsräte und alle ähnlichen grundsätzlich besonders flüchtlingsfreundlichen Organisationen mit am HFK-Tisch säßen, drohe in Bezug auf die in der HFK verhandelten Fälle die öffentliche Kritik stiller zu werden. Zu Fällen, über die man unter Verschwiegenheitspflicht mitentscheide, könne man sich schon aus strukturellen Gründen weniger ausführlich und offensiv äußern. Der Hypothese des Autors, dass sich dies auch auf die Vernehmbarkeit der öffentlichen Kritik an der asylpolitischen Rechtslage und Praxis insgesamt auswirken könnte, widersprachen die meisten befragten NRO-Experten zwar vehement, es wurde hier aber ohne Zweifel ein wunder und intern kontroverser Punkt berührt. Ein weiterer NRO-Vertreter äußerte die Ansicht, vermutlich sei es nicht die schlechteste Arbeitsteilung, wenn es NROs innerhalb und außerhalb der HFKs gebe, die unterschiedliche strategische Schwerpunkte in ihrer Arbeit setzten.

Potenziell noch heikler wird es dort, wo sich die HFK-Arbeit und andere Arbeitsbereiche von nichtstaatlichen Entsendeorganisationen in widersprüchlicher bis kontraproduktiver Weise berühren: So sind insbesondere die AWO und einige Re- 
gionalverbände von Caritas und Diakonischem Werk in der Beratung ${ }^{40}$ und bei Hilfen für sogenannte freiwillige Rückkehrer ${ }^{41}$ engagiert, für die sie öffentliche Mittel aus dem Europäischen Rückkehrfonds ${ }^{42}$ und teils auch von den Landesregierungen erhalten und teilweise ${ }^{43}$ weitergeben. In drei Interviews mit HFK-Mitgliedern aus verschiedenen Bundesländern wurde eingestanden, dass (oft entgegen dem Wortlaut der Regelungen zu herkunftslandbezogenen Sachverhalten) die durch Rückkehrhilfen verbesserte Situation im Herkunftsland in den HFKs entscheidungsrelevant werden kann. Einerseits kann hier - und das geschieht im internen Diskurs offenbar teils ganz massiv - die Frage nach der (nach Herkunftsland, hiesigem Anbieter und dortigem Kooperationspartner offenbar ganz erheblich variierenden) Seriosität und Wirksamkeit der entsprechenden Rückkehrhilfen gestellt werden (vgl. hierzu etwa Dünnwald 2008). Eine Antwort auf diese Frage kann an dieser Stelle nicht gegeben werden. Wohl aber auf die andererseits noch gravierendere danach, ob ein gleichzeitiges Engagement in HFK und Rückkehrhilfen nicht zu Befangenheit und damit einer Einschränkung der Legitimation der Mitwirkung führen kann - dies ist angesichts der gerade auch finanziellen Interessenkonflikte ohne Zweifel gegeben. Betont werden muss an dieser Stelle allerdings, dass nur eine Minderheit der in den HFKs vertretenen Organisationen (bzw. ihrer Landes- und Regionalverbände) in der Rückkehrarbeit aktiv ist. Zur Verteidigung des einschlägigen Engagements wurde überdies verschiedentlich angeführt, dass die allgemeine Flüchtlingsberatung (gerade auch von HFK-Kandidaten) ohne dieses vielerorts gar nicht

40 Zur Illustration dieser Arbeit sei hier ein typisches Faltblatt zitiert: „Wir informieren, beraten und entwickeln mit Ihnen im ergebnisoffenen Gespräch eine Perspektive für eine Rückkehr in Würde. [...] Eine Rückkehr bedeutet vieles: Sie können sich wieder in Ihrer Muttersprache ausdrücken, im vertrauten Kulturkreis leben und Ihre Freunde und Verwandte wiedersehen." Besonders umstritten ist innerhalb der Community, wer unter den Anbietern zu einer wirklich ergebnisoffenen Beratung überhaupt willens und in der Lage ist. Zu den üblicherweise offerierten Angeboten zählen neben einer Klärung der Perspektiven in Deutschland Hilfen bei der Existenzgründung im Herkunftsland, berufsbildende Schulungen mit mehr oder weniger Umfang und Bezug zur Situation dort, und Einmalzahlungen für Hausrat o. Ä. Gemäß den Ausschreibungsbedingungen „werden [...] vor allem Projekte für Rückkehrer/Rückkehrwillige aus bzw. Projekte in solchen Staaten [gefördert], die aus rückkehrpolitischer Sicht für Deutschland derzeit von besonderer Bedeutung sind. Hierbei handelt es sich [u.a.] um folgende Staaten: Afghanistan, Algerien, Äthiopien, China, Cote d'Ivoire, Irak, Iran, Jordanien, Kosovo, Kuba, Libanon, Nigeria, Pakistan, Russische Förderation [sic!], Sierra Leone, Somalia und Syrien.“ (BAMF 2010: 3)

41 Laut den Angaben von mehreren Interviewten sind die Fälle, in denen eine Freiwilligkeit jenseits akuten Abschiebedrucks vorliegt, eine winzige Minderheit.

42 Holzberger (2003: 113) zufolge trug Deutschland zum Vorgängerfonds des gegenwärtigen als größter Geldgeber rund ein Viertel bei.

43 Umstritten ist insbesondere die Praxis einiger Anbieter, eine unabhängig von den konkreten Leistungen an die Flüchtlinge erhobene Fallpauschale einzubehalten. 
mehr finanzierbar sei. Als aufgeschlossener Partner der staatlichen Seite zu gelten kann so zur (zumindest gefühlten) praxeologischen Notwendigkeit werden.

Schließlich kann es aus der HFK-Praxis auch zu Anstößen für die Flüchtlingsarbeit vor Ort kommen. So rät ein interviewter Kirchenvertreter den Kirchengemeinden, von der Konzentration auf die Kritik am Staat wegzukommen und mehr für das Erschließen der Integrationspotenziale der Gesellschaft zu tun, etwa Unternehmer in der Gemeinde zu suchen, die den Antragstellern Arbeit geben, und so die Chancen des Härtefallantrags zu erhöhen: „Der ständige Verweis auf die Härten des Staates trägt nur begrenzt weit.“

\section{Fazit: Feigenblatt oder i-Tüpfelchen?}

Die Arbeit der Härtefallkommissionen erweist sich als Feld, auf dem die systematische Variation vergleichsweise schwach ausgeprägt ist und dementsprechend qualitative Methoden wie Experteninterviews und teilnehmende Beobachtungen ertragreicher ausfallen als quantifizierende Analysen. Dennoch lassen sich folgende Muster erkennen: Wo die wirtschaftliche Lage Flüchtlingen eigene Beiträge zum Lebensunterhalt ceteris paribus leichter macht, steigen deren Chancen auf eine Aufenthaltserlaubnis via Härtefallantrag. Umfangreiche Listen von Nichtbefassungsund Regelausschlussgründen haben den gegenteiligen Effekt. Die Präsenz flüchtlingsfreundlicher Nichtregierungsorganisationen in den HFKs erhöht vor allem die Wahrscheinlichkeit, dass ein Antrag beraten wird, in nur geringem Ausmaß zudem seine Erfolgschancen. Linke Landesregierungen laden tendenziell ein breiteres Spektrum an zivilgesellschaftlichen Akteuren, insbesondere auch Flüchtlings- und Menschenrechtsorganisationen, in die HFKs ein und setzen niedrigere Mehrheitsschwellen. Im Ergebnis sind die Erfolgsaussichten der Antragsteller in diesen Ländern aber nicht höher, sondern geringer. Bürgerliche Landesregierungen beschränken sich dagegen häufig auf die Inklusion von Vertretern der Kirchen und der Wohlfahrtsverbände, geben dafür aber mehr Ersuchen der HFKs statt, die hier zudem öfter einstimmig beschlossen werden. Insbesondere für sie besteht der Reiz der HFK-Einrichtung und die besondere Konstruktion der Kooptation nichtstaatlicher Mitglieder darin, dass die HFK zugleich, wenn auch in verschiedenen Arenen, als Feigenblatt und i-Tüpfelchen fungieren kann. So bietet sie etwa Hardlinern unter den Innenministern die Chance, beim Vortrag vor einer Kirchengemeinde im eigenen Wahlkreis zu argumentieren, dass das deutsche Aufenthaltsrecht zugegebenermaßen gewisse Härten produziere, die HFKs aber für eben deren Korrektur eingerichtet worden seien und man den kirchlichen (und sonstigen nichtstaatlichen) Vertretern darin für das Einbringen ihrer spezifischen humanitären Kompetenz herzlich 
dankbar sei. An anderer Stelle können sie dagegen auf das Vetorecht der obersten Landesbehörde verweisen und betonen, dass eine zu großzügige Praxis der HFKs nicht geduldet werde. Erhellend ist als Synthese dazu folgendes Zitat aus dem Tätigkeitsbericht der bayerischen HFK für 2006/07:

„Die Glaubwürdigkeit der staatlichen Rückführungspolitik nimmt keinen Schaden, wenn in begründeten Einzelfällen ein humanitärer Ausgleich erfolgt. Vielmehr unterstreicht dies nachdrücklich, dass rechtliche Spielräume ausgeschöpft werden, wenn es sie tatsächlich gibt, und dient somit einer höheren Akzeptanz aufenthaltsbeendender Maßnahmen im Übrigen." (Geschäftsstelle der Härtefallkommission in Bayern 2007: 9)

In allen fünf näher betrachteten Ländern, inzwischen sogar im zeitweise stark polarisierten Hessen, ist ein großer HFK-Korpsgeist greifbar, identifizieren sich die interviewten Mitglieder stark mit ihrer Kommission und der jeweiligen Arbeitsweise. Auch scheint die Fallarbeit mit den Jahren routinierter und einhelliger geworden zu sein. Persönliche Haltungen bestimmen das Entscheidungsverhalten der Mitglieder dabei oft stärker als die entsendende Stelle. Öffentlichkeitswirksam konfliktiv wurde die HRK-Arbeit bislang nur in Einzelfällen.

Innerhalb der NROs und gelegentlich auch zwischen diesen wird die Teilnahme an den HFKs kontrovers diskutiert, weil ihre offensichtlichen Erfolge (bislang mehr als 6000 erteilte Aufenthaltstitel) dagegen abgewogen werden müssen, dass dieses gnadenrechtliche Element von staatlicher Seite als starkes Legitimationsargument für das humanitäre Aufenthaltsrecht als Ganzes, das die NROs weiterhin kritisieren, verwendet werden kann. Zudem steht zu vermuten, dass in Ländern, in denen praktisch die gesamte einschlägige NRO-Community in der HFK vertreten ist, die öffentliche Kritik am rechtlichen Status quo und dessen Anwendung auf einzelne Fälle leiser wird. Während die staatliche Seite also gut daran tut, NROs hinzuzuziehen und deren spezifische Kompetenzen möglichst noch offener als bislang zu nutzen, ist den NROs eine Reflexion über ihre Arbeitsteilung (sowie ggf. über ihr paralleles Engagement in HFKs und der Rückkehrberatung) anzuraten.

Jenseits einer streng verfassungslegalistischen Argumentationslinie kaum rechtfertigen lässt sich die Variation der Nichtbefassungs- und Regelausschlussgründe in den Landes-HFK-Gesetzen bzw. -Verordnungen, aus der für die betroffenen Flüchtlinge eine Postleitzahlen-Lotterie ihrer Antragschancen erwächst. Auch ein gnadenrechtliches Instrument sollte nicht willkürlich eingesetzt werden, und die üblichen Begründungen für föderale Vielfalt greifen hier nicht. Neben einer Angleichung dieser Regelungen, die nichtsdestoweniger den Kommissionen einen Ermessensspielraum belässt und auch im Kontext europäischer Harmonisierungen zu 
sehen ist, ${ }^{44}$ ist den Landesregierungen und -parlamenten zu raten, dem Willkür- und Missbrauchsverdacht vorzubeugen, der durch die in manchen Ländern praktizierte Antragstellung direkt bei einzelnen Mitgliedern entstehen kann. Zudem sollten sie als staatlicherseits nominierte HFK-Mitglieder selbstbewusste Persönlichkeiten einsetzen und diese dann auch gewähren zu lassen. Dann können die Härtefallkommissionen durchaus als Feigenblatt und i-Tüpfelchen zugleich fungieren.

Darüber hinaus sollte die hier vorgelegte Untersuchung gezeigt haben, dass die politikwissenschaftliche Befassung mit Institutionen zur Indienstnahme zivilgesellschaftlicher Kompetenz und Legitimation für staatliches Problemlösungshandeln den typischen Problemlösungsbias der Governance-Literatur (vgl. Blumenthal 2005: 1172) vermeiden sollte, indem sie für Fragen der Macht und Herrschaft sensitiv bleibt (vgl. hierzu Mayntz 2004: 74), wie sie etwa in den theoretischen Ansätzen der vergleichenden Staatstätigkeitsforschung prominenter Berücksichtigung finden.

\section{Literatur}

Allenberg, Nele, 2010: Aufenthaltserlaubnis aufgrund einer Härtefallentscheidung, in: Jürgen Blechinger/Vera Weißflog (Hrsg.), Das neue Zuwanderungsrecht. Praxishandbuch zur rechtssicheren Umsetzung der aktuellen nationalen und europäischen Vorschriften, Merching, Abs. 5.12 der Loseblattsammlung, 16 S. (Stand: 2/10).

Apitzsch, Ursula, 2003: Individuelle Bearbeitung sozialer Unsicherheit in prekären Migrationsverläufen: Self employment als biographischer Prozess, in: Jutta Allmendinger (Hrsg.), Entstaatlichung und Soziale Sicherheit. Verhandlungen des 31. Kongresses der Deutschen Gesellschaft für Soziologie in Leipzig 2003, Opladen, 219-235.

Benz, Arthur, 2004: Governance - Modebegriff oder nützliches sozialwissenschaftliches Konzept?, in: Arthur Benz (Hrsg.), Governance - Regieren in komplexen Regelsystemen, Wiesbaden, 11-28.

Berger, Michael B., 2010: Ein Streit ums Maß der Humanität, Hannoversche Allgemeine Zeitung, 29.9.2010, 5.

Blumenthal, Julia von, 2005: Governance - eine kritische Zwischenbilanz, in: Zeitschrift für Politikwissenschaft 15 (4), 1149-1180.

44 Nach der de facto-Suspendierung des Dublin II-Systems in Bezug auf Griechenland durch das Bundesverfassungsgericht ist auch hier der Reformdruck noch einmal gestiegen. 
Brown, Garrett W., 2010: The Laws of Hospitality, Asylum Seekers and Cosmopolitan Right: A Kantian Response to Jacques Derrida, in: European Journal of Political Theory 9 (3), 308-327.

Buckel, Sonja/Wissel, Jens, 2010: State Project Europe: The Transformation of the European Border Regime and the Production of Bare Life, in: International Political Sociology 4 (1), 33-49.

Bundesamt für Migration und Flüchtlinge (BAMF), 2010: Aufforderung des Bundesamtes für Migration und Flüchtlinge zur Einreichung von Anträgen auf Gewährung einer Zuwendung aus dem Europäischen Rückkehrfonds für das Förderjahr 2010, Nürnberg.

Butterwegge, Christoph, 2009: Globalisierung als Spaltpilz und sozialer Sprengsatz. Weltmarktdynamik und ,Zuwanderungsdramatik“ im postmodernen Wohlfahrtsstaat, in: Christoph Butterwegge/Gudrun Hentges (Hrsg.), Zuwanderung im Zeichen der Globalisierung. Migrations-, Integrations- und Minderheitenpolitik, Opladen (4. Aufl.), 55-102.

Cable, Vincent, 1995: The Diminished Nation-State: A Study in the Loss of Economic Power, in: Daedalus 124 (2), 23-53.

Dünnwald, Stephan, 2008: Angeordnete Freiwilligkeit. Zur Beratung und Förderung freiwilliger und angeordneter Rückkehr durch Nichtregierungsorganisationen in Deutschland, München.

Ephross, Paul H./Vassil, Thomas V., 1988: Groups that Work: Structure and Process, New York.

Gäbel, Ulrike/Ruf, Martina/Schauer, Maggie/Odenwald, Michael/Neuner, Frank, 2006: Prävalenz der Posttraumatischen Belastungsstörung (PTSD) und Versuch der Erfassung der Störung im Rahmen der Asylverfahrenspraxis, in: Zeitschrift für Klinische Psychologie und Psychotherapie 35 (1), 12-20.

Geschäftsstelle der Härtefallkommission in Bayern, 2007: Bericht über die Tätigkeit der Härtefallkommission des Freistaates Bayern 2006/2007, München.

Geschäftsstelle der Härtefallkommission in Bayern, 2009: Bericht über die Tätigkeit der Härtefallkommission des Freistaates Bayern 2008, München.

Geschäftsstelle der Härtefallkommission in Hessen, 2011: Bericht über die Tätigkeit der Härtefallkommission des Landes Hessen 2010, Wiesbaden.

Härtefallkommission beim Innenministerium Baden-Württemberg, 2009: Dritter Tätigkeitsbericht der Härtefallkommission beim Innenministerium BadenWürttemberg. Berichtszeitraum 1. Januar bis 31. Dezember 2008, Stuttgart.

Hildebrandt, Achim/Wolf, Frieder, 2008: Die Potenziale des Bundesländervergleichs, in: Achim Hildebrandt/Frieder Wolf (Hrsg.), Die Politik der Bundesländer. Staatstätigkeit im Vergleich, Wiesbaden, 11-20. 
Holzberger, Mark, 2003: Die Harmonisierung der europäischen Flüchtlingspolitik, in: Christoph Butterwegge/Gudrun Hentges (Hrsg.), Zuwanderung im Zeichen der Globalisierung. Migrations-, Integrations- und Minderheitenpolitik, Opladen, 111-122.

Holzer, Thomas/Schneider, Gerald, 2002: Asylpolitik auf Abwegen. Nationalstaatliche und europäische Reaktionen auf die Globalisierung der Flüchtlingsströme, Opladen.

Hunt, Lisa, 2008: Women Asylum Seekers and Refugees. Opportunities, Constraints and the Role of Agency, Social Policy and Society 7 (3), 281-292.

Mayntz, Renate, 2004: Governance im modernen Staat, in: Arthur Benz (Hrsg.): Governance - Regieren in komplexen Regelsystemen, Wiesbaden, 65-76.

Menz, Georg, 2009: The Political Economy of Managed Migration. Nonstate Actors, Europeanization, and the Politics of Designing Migration Policies, Oxford. Mills, Theodore M., 1967: The Sociology of Small Groups, London.

Muy, Sebastian/Sulimma, Stephen, i. E.: Strukturelle Rahmenbedingungen sozialer Arbeit im Handlungsfeld Flucht und Migration, in: Netzwerk MiRA (Hrsg.), Kritische Migrationsforschung. Da kann ja jedeR kommen, Berlin.

Puumala, Eeva/Pehkonen, Samu, 2010: Coporeal Choreographies between Politics and the Political: Failed Asylum Seekers Moving from Body Politics to Bodyspaces, in: International Political Sociology 4 (1), 50-65.

Ruback, Timothy J., 2010: 'Let Me Tell the Story Straight On': Middlemarch, Process-Tracing Methods and the Politics of Narrative, in: British Journal of Politics and International Relations 12 (4), 477-497.

Sader, Manfred, 1976: Psychologie der Gruppe, München.

Schmidt, Manfred G., 1980: CDU und SPD an der Regierung. Ein Vergleich ihrer Politik in den Ländern, Frankfurt a. M.

Schmidt, Manfred G./Ostheim, Tobias/Siegel, Nico A./Zohlnhöfer, Reimut, 2007: Der Wohlfahrtsstaat, Wiesbaden.

Seifer, Kerstin, 2009: Governance als Einfluss-System. Der politische Einfluss von NGOs in asymmetrisch strukturierten Interaktionsarrangements, Wiesbaden.

Simmel, Georg, 1908: Soziologie. Untersuchungen über die Formen der Vergesellschaftung.

Töller, Annette Elisabeth/Dittrich, Marcus, 2010: Die Privatisierung des Maßregelvollzugs. Die deutschen Bundesländer im Vergleich, polis-Arbeitspapier Nr. 68/2010, Hagen.

Wais, Edgar, 2010: Bleiberecht für Ausländer ,gnadenhalber ' - Last oder Gewinn für die Landkreise?, in: Der Landkreis 80 (6), 254-257. 
Weber, Christoph, 2005: Arbeit der Härtefallkommission beim Innenministerium des Landes Nordrhein-Westfalen, in: Zeitschrift für Ausländerrecht und Ausländerpolitik 25 (6), 203-204.

Wolf, Frieder, 2006: Die Bildungsausgaben der Bundesländer im Vergleich. Welche Faktoren erklären ihre beträchtliche Variation?, Münster.

Wolf, Frieder/Zohlnhöfer, Reimut, 2009: Investing in Human Capital? The Determinants of Private Education Expenditure in 26 OECD Countries, in: Journal of European Social Policy 19 (3), 230-244.

Korrespondenzanschrift:

Dr. Frieder Wolf

Ruprecht-Karls-Universität Heidelberg

Institut für Politische Wissenschaft

Bergheimer Straße 58

69115 Heidelberg

wolf@uni-hd.de 